\title{
Pancreatic cancer is marked by complement-high blood monocytes and tumor-associated macrophages
}

\author{
Samantha B Kemp ${ }^{1}$, Nina G Steele², Eileen S Carpenter ${ }^{3}$, Katelyn L Donahue ${ }^{4}$, Grace G Bushnell ${ }^{5}$, Aaron H Morris ${ }^{5}$, \\ Stephanie The ${ }^{6}$, Sophia M Orbach ${ }^{5}$, Veerin R Sirihorachai ${ }^{4}$, Zeribe C Nwosu', Carlos Espinoza ${ }^{8}$, Fatima Lima ${ }^{8}$, \\ Kristee Brown ${ }^{8}$, Alexander A Girgis ${ }^{8}$ (1), Valerie Gunchick ${ }^{9}$, Yaqing Zhang ${ }^{8}$, Costas A Lyssiotis ${ }^{7,12}$ (D, Timothy L Frankel ${ }^{8,12}$, \\ Filip Bednar ${ }^{8,12}$ (D), Arvind Rao $5,6,10,11,12$, Vaibhav Sahai ${ }^{9}$ (D), Lonnie D Shea ${ }^{5}$, Howard C Crawford ${ }^{4,7,12}$ (1), \\ Marina Pasca di Magliano $2,4,8,12$ (1)
}

Pancreatic ductal adenocarcinoma (PDA) is accompanied by reprogramming of the local microenvironment, but changes at distal sites are poorly understood. We implanted biomaterial scaffolds, which act as an artificial premetastatic niche, into immunocompetent tumor-bearing and control mice, and identified a unique tumor-specific gene expression signature that includes high expression of C1qa, C1qb, Trem2, and Chil3. Singlecell RNA sequencing mapped these genes to two distinct macrophage populations in the scaffolds, one marked by elevated C1qa, C1qb, and Trem2, the other with high Chil3, Ly6c2 and Plac8. In mice, expression of these genes in the corresponding populations was elevated in tumor-associated macrophages compared with macrophages in the normal pancreas. We then analyzed single-cell RNA sequencing from patient samples, and determined expression of C1QA, C1QB, and TREM2 is elevated in human macrophages in primary tumors and liver metastases. Single-cell sequencing analysis of patient blood revealed a substantial enrichment of the same gene signature in monocytes. Taken together, our study identifies two distinct tumorassociated macrophage and monocyte populations that reflects systemic immune changes in pancreatic ductal adenocarcinoma patients.

DOI 10.26508/lsa.202000935 | Received 14 October 2020 | Revised 12 March 2021 | Accepted 12 March 2021 | Published online 29 March 2021

\section{Introduction}

Pancreatic ductal adenocarcinoma (PDA) is a lethal malignancy with a dismal 5-yr survival rate of only $10 \%$ (Siegel et al, 2020). PDA is characterized by an abundant, fibroinflammatory stroma. From the onset of carcinogenesis, the immune response to pancreatic cancer results in an immunosuppressive tumor microenvironment (TME) (Clark et al, 2007). Myeloid cells are abundant and heterogeneous within the PDA TME and are a key driver of an immune suppressive microenvironment (Mitchem et al, 2013; Stromnes et al, 2014; Zhang et al, 2017; Zhu et al, 2014, 2017). The primary tumor and metastatic sites are both characterized by tumor cell evasion of the immune response (Hanahan \& Weinberg, 2011; Gonzalez et al, 2018). However, systemic alteration of the immune system by the primary tumor remains poorly understood. Although the stochastic nature of metastasis greatly limits our ability to study the systemic responses to the primary tumor, recent advances in biomaterials engineering provide a novel opportunity to evaluate systemic response to PDA through the use of polycaprolactone scaffolds.

Biomaterial scaffolds have been used as a synthetic premetastatic niche in breast and pancreas cancer models (Azarin et al, 2015; Rao et al, 2016; Aguado et al, 2017; Bushnell et al, 2019, 2020). Implantation of biomaterial scaffolds initially causes a foreign body response in both control and tumor-bearing mice. Over time, scaffolds in tumor-bearing mice develop a microenvironment supportive of cancer cell colonization. Scaffolds have been used more recently as a tool to obtain gene signatures that are predictive of disease and recurrence in mouse models of breast cancer and multiple sclerosis, an application of high clinical relevance (Oakes et al, 2020; Morris et al, 2020a, 2020b). Scaffolds, unlike the blood, allow for analysis of tissue-based immune response at distal sites.

In this study, we used engineered polymer scaffolds implanted into immune competent mice with orthotopic pancreatic tumors to generate an immune gene signature associated with pancreatic cancer. We found fundamental differences in the gene expression

\footnotetext{
${ }^{1}$ Departments of Molecular and Cellular Pathology, University of Michigan, Ann Arbor, MI, USA ${ }^{2}$ Cell and Developmental Biology, University of Michigan, Ann Arbor, MI, USA ${ }^{3}$ Internal Medicine, Division of Gastroenterology, University of Michigan, Ann Arbor, MI, USA ${ }^{4}$ Cancer Biology, University of Michigan, Ann Arbor, MI, USA ${ }^{5}$ Biomedical Engineering, University of Michigan, Ann Arbor, MI, USA ${ }^{6}$ Computational Medicine and Bioinformatics, University of Michigan, Ann Arbor, MI, USA ${ }^{7}$ Molecular and Integrative Physiology, University of Michigan, Ann Arbor, MI, USA ${ }^{8}$ Surgery, University of Michigan, Ann Arbor, MI, USA ${ }^{9}$ Internal Medicine, Division of Hematology and Oncology, University of Michigan, Ann Arbor, MI, USA ${ }^{10}$ Radiation Oncology, University of Michigan, Ann Arbor, MI, USA ${ }^{11}$ Biostatistics, University of Michigan, Ann Arbor, MI, USA ${ }^{12}$ Rogel Cancer Center, University of Michigan, Ann Arbor, MI, USA
} 
of cellular infiltrates derived from scaffolds in tumor-bearing versus non-tumor mice, with a tumor-specific signature including Chil3, Trem2, C1qa, and C1qb. Single-cell RNA sequencing identified changes primarily in macrophage gene expression and revealed two distinct populations of macrophages that were unique to tumor-bearing animals. Whereas one macrophage population expressed Chil3, Ly6c2, and Plac8, the other expressed Trem2 and complement components C1qa and C1qb (complement-high macrophage). The complement-high macrophage population was present in primary tumors from mice and PDA patients, metastatic liver lesions, and expression of $C 1 Q A, C 1 Q B$, and TREM2 was elevated in tumors and blood from human PDA patients. Thus, we defined two-distinct systemically altered macrophage populations associated with PDA.

\section{Results}

\section{Biomaterial scaffolds harbor an immune-dense microenvironment in response to an orthotopic model of PDA}

To understand the systemic immune changes in PDA, we first assessed the immune infiltration in the liver and peripheral blood of tumor-bearing animals compared with controls. We used an orthotopic syngeneic model using 7940b cells (Long et al, 2016; Zhang et al, 2017), derived from a pure C57BL/6J (BL/6) version of the $\mathrm{LSL}_{-K \mathrm{ras}^{\mathrm{G12D/+}} \text {; LSL-Trp53 }}^{\mathrm{R172H/+}}$; Pdx1-Cre (KPC) genetically engineered mouse model of pancreatic cancer (Hingorani et al, 2005). We orthotopically implanted 7940b cells into the pancreas and performed mass cytometry (CYTOF) analysis of the resulting tumors. We found that livers and PBMCs from tumor-bearing mice had an increase in myeloid cells preceding the outgrowth of metastases, similar to previous reports (Rhim et al, 2012; Sanford et al, 2013; Li et al, 2018; Lee et al, 2019) (Fig S1A and B).

The immune cell changes in the blood and liver of tumor-bearing mice provided evidence of a systemic immune response to the tumor. We next used biologically inert polycaprolactone scaffolds to further study how tumors alter the systemic immune response in pancreatic cancer (Fig S1C). We first implanted scaffolds subcutaneously into BL/ 6 mice. 1 wk later 7940b (BL/6) cells were orthotopically transplanted into the pancreas, followed by removal of scaffolds weekly over the course of 4 wk (Fig S1D). Control mice had subcutaneous scaffold implantation, followed by mock orthotopic surgery. We isolated RNA from control and tumor-bearing scaffolds and used a qRT-PCR array (OpenArray, OA) to assess a panel of 632 mouse inflammatory genes and 16 reference genes (Fig S1D). Interestingly, earlier control time points were more similar to tumorbearing scaffolds, suggesting a foreign body response that subsides over time (Fig S1D). Based on the inflammatory gene changes over time in the scaffold infiltrate we performed our subsequent experiments at a fixed 3-wk time point. We next orthotopically transplanted $7940 \mathrm{~b}$ cells into $\mathrm{BL} / 6$ mice, implanted scaffolds $1 \mathrm{wk}$ later and then harvested the scaffolds after 3 wk (Fig 1A). Scaffolds from control and tumor-bearing animals were examined by immunofluorescence staining to determine which cell populations colonized the scaffold. Epithelial cells $\left(\mathrm{CK} 19^{+}\right)$were identified only in tumor bearing mice, suggesting colonization by PDA cells (Fig 1B). Furthermore, we observed a stromal response in the tumor-bearing scaffold, characterized by accumulation of fibroblasts (alphasmooth muscle actin [ $\alpha$-SMA]) (Fig 1B).

To determine whether the immune response in the scaffold was distinct in tumor-bearing versus healthy mice, we performed CyTOF using a panel of immune markers (Table S1). Visualization of the scaffold infiltrate by t-distributed stochastic neighbor embedding (t-SNE) in control and tumor-bearing animals revealed an abundant stromal response in both, with most of the infiltrate comprising various myeloid subsets, including macrophage subsets and myeloid-derived suppressor cells (MDSCS) (Fig 1C). Whereas there was no difference in total myeloid $\left(C D 45^{+}\right.$CD11 $\left.{ }^{+}\right)$, MDSC (CD11b ${ }^{+}$ Ly- $\left.6 \mathrm{G}^{+} \mathrm{Ly}-6 \mathrm{C}^{+}\right)$, or total macrophage $\left(\mathrm{CD} 11 \mathrm{~b}^{+} \mathrm{F} 4 / 80^{+}\right)$infiltration expressed as percentage of total cells, we observed an upward trend for specific macrophage populations (CD11b ${ }^{+} \mathrm{F} 4 / 80^{+} \mathrm{CD} 206^{+}$; $\left.\mathrm{CD}_{11 \mathrm{~b}^{+}} \mathrm{F} 4 / 80^{+} \mathrm{PD}-\mathrm{L}^{+}\right)$in scaffolds from tumor-bearing animals compared with controls, similar to the increase in macrophages in the tumor-bearing liver (Figs S1A and E and 1D). In addition, tumorbearing scaffolds had a higher proportion of endothelial cells $\left(\mathrm{CD}_{4} 5^{-} \mathrm{PECAM}^{+}\right)$and fibroblasts $\left(\mathrm{CD} 45^{-} \mathrm{PDGFR}^{+}\right)$than control scaffolds (Fig 1E). Finally, we analyzed the adaptive immune populations, and observed that tumor-bearing scaffolds had fewer total $\mathrm{T}$ cells $\left(\mathrm{CD} 45^{+} \mathrm{CD} 3^{+}\right)$, and fewer $\mathrm{CD} 8^{+} \mathrm{T}$ cells compared with control (Figs S1E and 1E). Thus, cell composition data suggested that the microenvironment at a distal site was altered in tumor-bearing mice.

\section{Identification of a pancreatic cancer-specific gene signature}

To understand the nature of the systemic changes in tumor bearing mice, we isolated RNA from scaffolds implanted in control and tumor-bearing mice and used the qRT-PCR inflammatory OA. Two computational approaches were used to assign numerical scores to the mice and distinguish healthy (black) from diseased (red) (Fig 2A) (Morris et al, 2020a). Unsupervised hierarchical clustering analysis revealed that tumor-bearing scaffolds (red) clustered separately from control scaffolds (black) at the gene expression level (Fig S2A and B). We further observed that, although the inflammatory signature of control scaffolds appeared rather uniform, there was distinct heterogeneity amongst tumor-bearing scaffolds from individual mice (Fig S2A). We then analyzed the data to define a unique 21 gene signature indicative of disease (Fig 2B). Tumorbearing scaffolds had lower expression of interferon $y$ (Ifng) and killer cell lectin like receptor G1 (Klrg1), markers of T-cell activation / effector T cells, and, conversely, up-regulation of coagulation factor II thrombin receptor (F2r), a marker of exhausted T cells (Wherry et al, 2007) (Fig 2B). In addition, tumor-bearing scaffolds had upregulation of chitinase3-like-3 (Chil3), a gene elevated in tumorassociated macrophages (TAMs) (Georgoudaki et al, 2016) (Fig 2B). Bulk RNA analysis provided an indication that the immune composition and functional status might be altered systemically in mice bearing pancreatic cancer.

To understand gene expression changes at a cellular level, we performed single-cell RNA sequencing on cells isolated from the scaffolds extracted from control and tumor-bearing mice. Using published lineage markers, we defined the captured cells (Elyada 
A

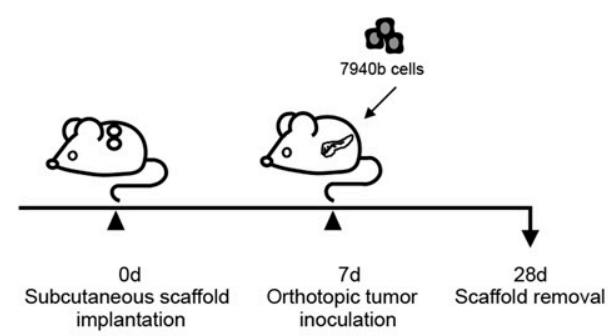

B

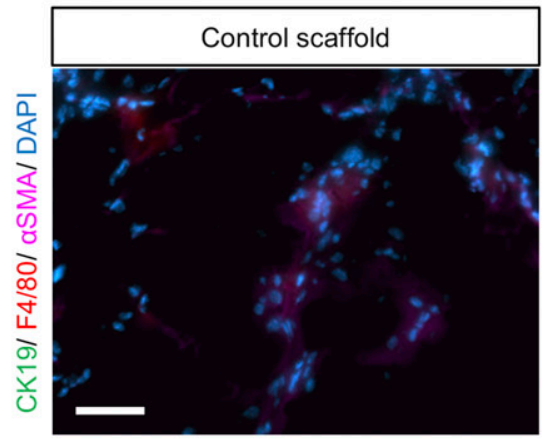

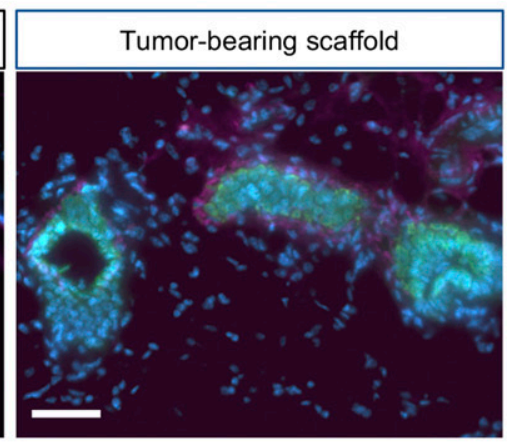

C
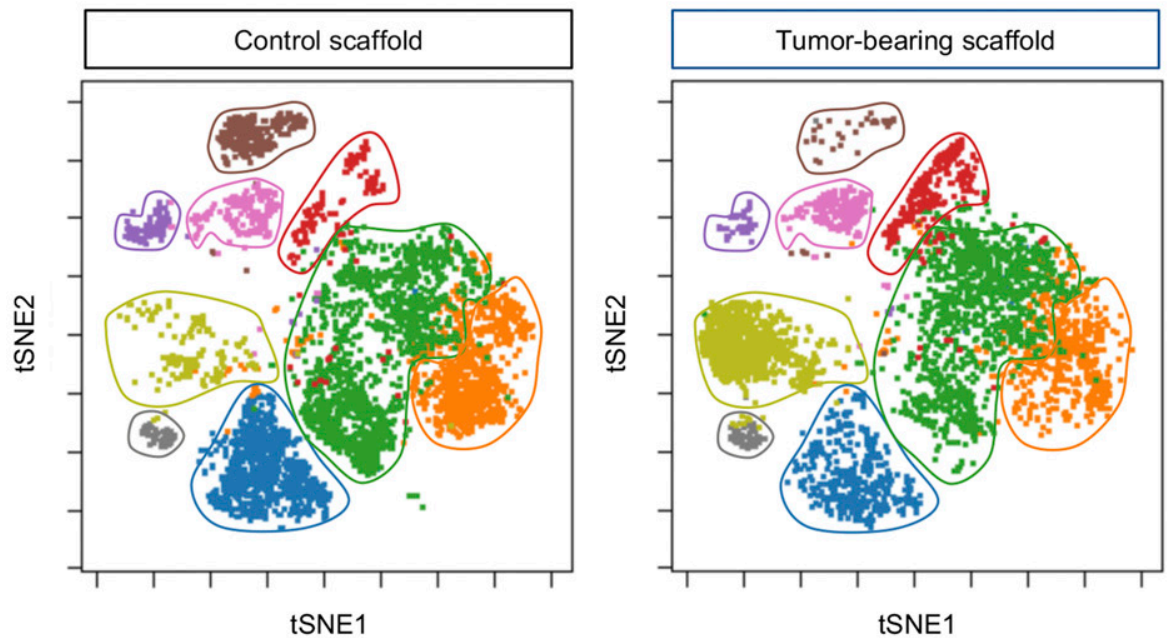

MDSCs (CD11 b $\left.\mathrm{b}^{+} \mathrm{Ly}-6 \mathrm{C}^{+} \mathrm{Ly}-6 \mathrm{G}^{+}\right)$

Macrophages (CD11 b+ F4/80+ Ly-6C+)

Macrophages (CD11 b+ F4/80+ PD-L1+)

Macrophages (CD11 b+ F4/80+ CD206 $\left.{ }^{+}\right)$

$\mathrm{CD}^{+} \mathrm{T}$ cells $\left(\mathrm{CD} 3^{+} \mathrm{CD} 8^{+}\right)$

$\mathrm{CD}^{+}+\mathrm{T}$ cells $\left(\mathrm{CD} 3^{+} \mathrm{CD}^{+}\right)$

NK cells $\left(C D 45^{+}\right.$NK1.1+)

Endothelial cells (CD45- PECAM-1+)

Fibroblasts (CD45- PDGFRa ${ }^{+}$)

tSNE1

E


Figure 1. Biomaterial scaffolds harbor an immune-dense microenvironment in response to an orthotopic model of PDA.

(A) Experimental scheme. Scaffolds were subcutaneously implanted as described in the Materials and Methods section. 7940b (BL/6) cells derived from the LSL$\mathrm{Kras}^{\mathrm{G12D/+}}$; LSL-Trp53 ${ }^{\mathrm{R} 172 \mathrm{H} /+}$; Pdx1-Cre (KPC) were orthotopically implanted into the pancreas. Scaffolds were harvested 3 wk post tumor cell inoculation. (B) Coimmunofluorescence of scaffolds from animals who underwent mock-surgery (left) compared with tumor-bearing (TB) mice (right). Tumor cells are marked by CK19 (green), macrophages by F4/80 (red), fibroblasts by aSMA (pink), and nuclei by DAPI (blue). Scale bars, $50 \mu \mathrm{m}$. (C) Representative t-SNE plots for the scaffold infiltrate from control and TB scaffolds. Identified populations include, myeloid-derived suppressor cells (blue), Ly-6C macrophages (orange), PD-L1 ${ }^{+}$macrophages (green), $\mathrm{CD}^{206^{+}}$macrophages (red), CD8 ${ }^{+} \mathrm{T}$ cells (purple), CD4 ${ }^{+} \mathrm{T}$ cells (brown), NK cells (pink), endothelial cells (grey), and fibroblasts (light green). (D) Manual gating of CyTOF results for macrophage subsets $\left(\mathrm{F} 4 / 80^{+} \mathrm{CD} 206^{+} ; \mathrm{F} 4 / 80^{+} \mathrm{PD}-\mathrm{L} 1^{+}\right)$in control scaffold $(n=8)$ compared with TB scaffold $(n=7-8)$. Results are plotted as percent of total myeloid cells $\left(\% \mathrm{CD} 11 \mathrm{~b}^{+}\right)$. Statistical significance was determined using two-tailed $t$ tests. Data presented as means \pm standard error (SEM) and $P<0.05$ was considered statistically significant. (E) Manual gating of CyTOF results for endothelial cells (CD45- PECAM1 $1^{+}$), fibroblasts (CD45- PDGFRa ${ }^{+}$) and CD8 ${ }^{+}$T cells $\left(\mathrm{CD}^{+} \mathrm{CD}^{+}\right)$in control Scaffold $(n=10)$ compared with TB scaffold $(n=10)$. Results are plotted as percent of total live singlets. Statistical significance was determined using two-tailed $t$ tests. Data presented as means \pm standard error (SEM) and $P<0.05$ was considered statistically significant.

Source data are available for this figure. 
A

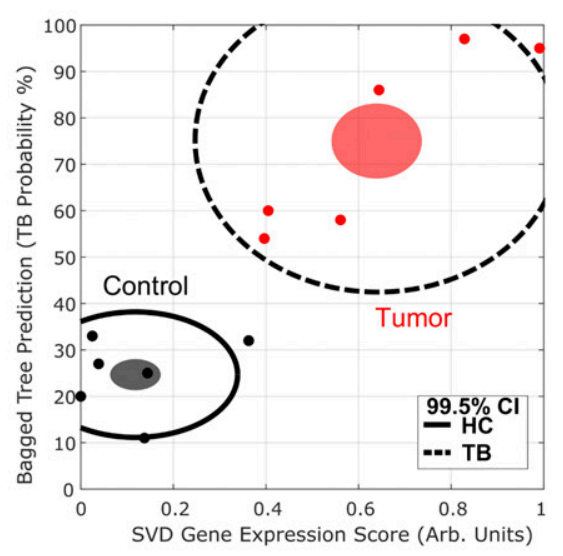

B

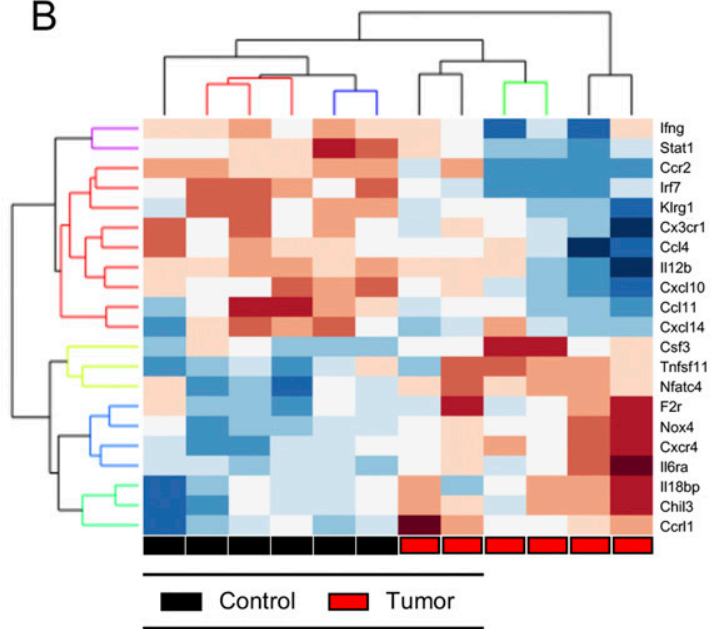

C

Scaffold single cell RNA sequencing

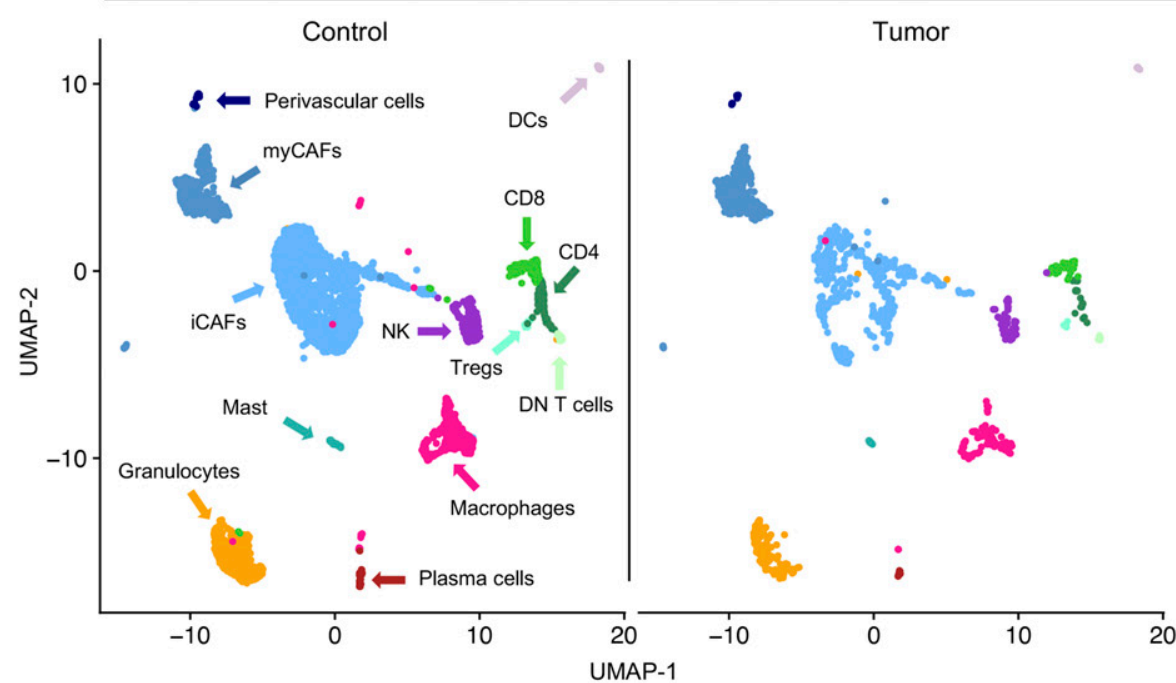

iCAFs

- Granulocytes

- myCAFs

- Macrophages

- NK cells

- CD8 T cells

- CD4 T cells

- Plasma cells

- Mast cells

- Perivascular cells

- DCs

DN T cells

- Tregs

D Scaffold single cell RNA sequencing

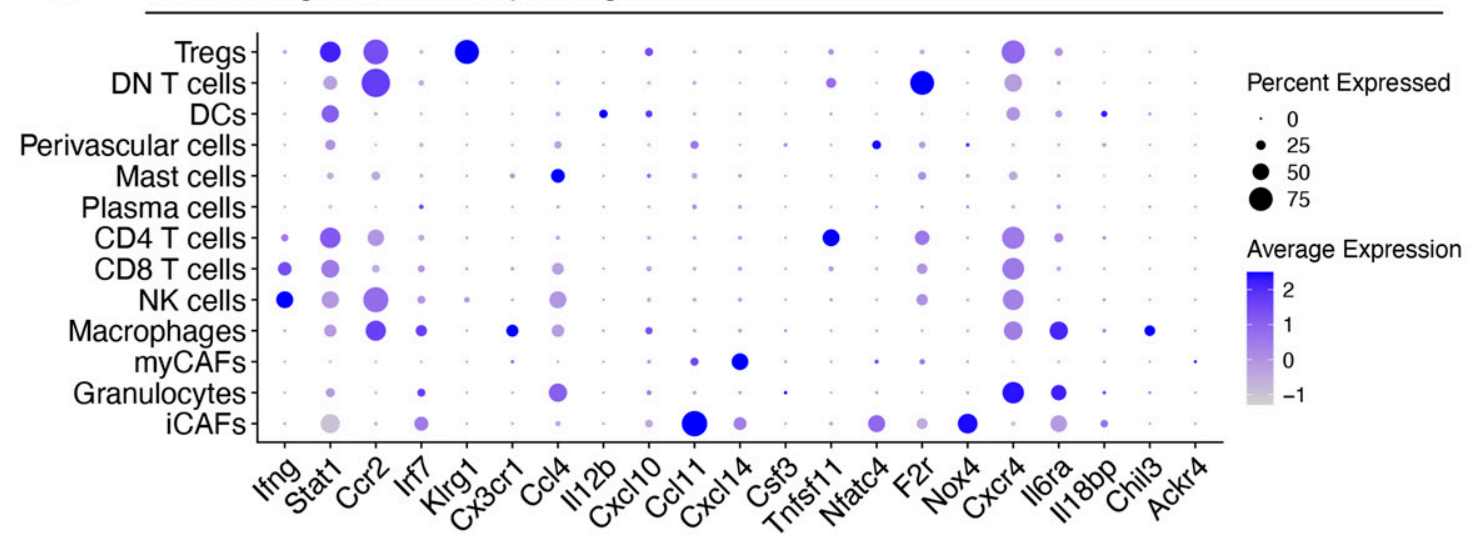

Figure 2. Identification of a pancreatic cancer-specific gene signature.

(A) Plot of Bagged Tree/singular value decomposition prediction produced from inflammatory gene OpenArray. Plot highlights the divergence of tumor-bearing (TB) scaffolds (red) from healthy control (HC) scaffolds (black). $n=6$ for control and $n=6$ for TB scaffolds. Each dot represents a single mouse. Black line indicates $99.5 \%$ confidence intervals. Filled ovals denote the mean for control (black) and TB (red) scaffolds for pooled control or TB scaffolds. (B) Hierarchical clustering and heat map of 21 inflammatory genes of interest in control $(n=6)$ scaffolds compared with TB scaffolds $(n=6)$. (C) UMAP visualization of control scaffold $(n=1)$ and TB $(n=1)$ scaffolds from an orthotopic mouse model of pancreatic cancer. (D) Dot plot shows average expression of scaffold signature in merged control and TB scaffold infiltrate. Size of dot represents percent expressed. Color of dot represents average expression. 
et al, 2019) (Fig S2C). We performed downstream analysis on all captured stromal cells, including cancer-associated fibroblast (CAF) subsets (myofibroblastic-CAFs [myCAF] and inflammatory-CAFs [iCAF]) (Ohlund et al, 2017), perivascular cells, NK cells, T-cell subsets (CD4, CD8, double-negative [DN] T cells, and regulatory T cells [Treg]), plasma cells, mast cells, DCs, and myeloid cell subsets (granulocytes and macrophages) (Fig 2C). Analysis of the scaffold gene profile further revealed cell type specific gene signatures (Fig 2D). Given the changes in myeloid cells and macrophages in the liver and blood of tumor-bearing mice (Fig S1A and B) we subsequently focused on the scaffold-associated macrophages (SAMs). We detected expression of Chil3 and Interleukin 6 Receptor (Il6ra) (Fig 2D), which have both been identified as playing a role in polarization of alternatively activated macrophages (Mauer et al, 2014; Roszer, 2015; Liou et al, 2017). By immunostaining, we detected an increase in $\mathrm{Ym}^{+}$(Chil3) cells in the livers of tumor-bearing compared with control mice, providing further evidence that changes in the immune cell component occur both in the scaffolds and in the natural metastatic site and precede overt metastasis development (Fig S2D and E).

\section{Identification of two distinct macrophage subsets in scaffold infiltrate}

We next compared the gene expression profile of SAMs in tumorbearing mice versus controls. The differentially expressed genes corroborated the scaffold signature with lower expression of interferon regulatory factor 7 (Irf7) and signal transducer and activator of transcription 1 (Stat1), as well as increased expression of Chil3 (Figs 3A-C and 2B and Table S2). In addition, tumor-bearing SAMs displayed a high expression of complement C1q A chain and $B$ chain (C1qa and C1qb) and triggering receptor expressed on myeloid cells 2 (Trem2), and a low expression of the major histocompatibility complexes (Cd74, H2-D1, H2-Aa, and H2-Eb1) compared with control SAMs (Fig $3 A$ and B). Thus, SAMs from tumorbearing mice at a distal site have distinct gene expression compared with controls. While C1qa, C1qb, and Trem2 are known drivers of alternatively activated macrophage polarization in a LPSinduced inflammation model (Turnbull et al, 2006; Benoit et al, 2012), little is known about their involvement in pancreatic cancer.

Using uniform manifold approximation and projection (UMAP) analysis on the SAMs we identified two transcriptionally distinct macrophage populations in the control and tumor-bearing scaffold infiltrate (Figs 3D and S3A). Unbiased analysis of the top genes defining each cluster identified C1qa, C1qb, and Trem2 as markers of the SAM 1 population, whereas Chil3, placenta associated 8 (Plac8), and $L y 6 C 2$ emerged as markers of SAM 2 (Figs S3B and $C$ and $3 E$ and F). SAM 1 also had high expression of Cd74, H2-Eb1, and H2-Aa (Fig S3D). Taken together, SAMs segregated into two main populations and have a different gene expression pattern in tumor-bearing mice compared with SAMs from healthy mice.

\section{Macrophages in mouse pancreatic tumors overexpress TREM2 and complement genes}

Having identified Chil3, Trem2, and the complement genes, C1qa and $C 1 q b$ as markers of SAMs in tumor-bearing mice, we next investigated whether these macrophage subsets also exist in primary tumors. To this end, we performed single-cell RNA sequencing on two primary mouse orthotopic PDA tumors. We identified populations of epithelial cells, acinar cells, fibroblasts, and six immune cell populations, including macrophages (Figs 4A and S4A). Compared with other immune cells, the macrophages in the primary tumor (i.e., TAMs) exclusively exhibited high expression of the SAMs signature genes (Chil3, Trem2, C1qa, and C1qb), whereas Plac8 and Ly6c2 were broadly expressed across cell types (Fig 4B). Unbiased clustering identified 2 distinct populations of macrophages in the primary tumor (Figs 4C and S4B). Similar to the SAMs, the TAMs in the primary tumor separated into two populations: one with high expression of Chil3, Plac8, and Ly6c2 (Chil-TAMs), and the other with high expression of C1qa, C1qb, and Trem2 (Cq-TAMs) (Fig 4D). ChilTAMs had higher expression of the inflammatory macrophage markers nitric oxide synthase 2 (Nos2) and tumor necrosis factor (Tnf) (Murray \& Wynn, 2011), whereas Cq-TAMs had higher expression of the alternatively activated macrophage markers Mrc1 and Cd163 (Roszer, 2015) (Fig S4C).

We then compared TAMs in orthotopic KPC tumors (Tumor) to normal mouse pancreas (N Panc) (Fig 4E). In both conditions, we detected Chil-TAMs, Cq-TAMs, and an additional population of macrophages (TAM) (Figs 4E and S4D). The expression of Chil-TAM and $\mathrm{Cq}$-TAM markers in the respective populations was elevated in orthotopic tumors compared with the normal pancreas (Fig 4F). By co-immunofluorescence we detected an increase in $\mathrm{Cq}-\mathrm{TAMs}\left(\mathrm{C}_{\mathrm{Cq}}{ }^{+}\right.$ $\left.\mathrm{F} 4 / 80^{+}\right)$in KPC tumors and KPC liver metastasis compared with the normal pancreas (Fig 4G). Taken together, we detected an increase in Cq-TAMs and an increase in the expression of Chil3, Trem2, C1qa, and $C 1 q b$ in the tumor compared with the normal pancreas.

We next compared macrophages from scaffolds with macrophages from orthotopic mouse tumors (Fig S4E) and plotted differentially expressed genes. We observed higher expression of Arg1, Il1a, and Rgs1 in macrophages from scaffolds compared with those from the primary tumor (Fig S4F). Thus, macrophages at the primary and distant sites are similar but retain distinct features. Thus, these two distinct macrophage populations (Chil-TAMs and Cq-TAMs) are prevalent both at the primary tumor and systemically in response to pancreatic cancer in mice.

We then sought to validate the presence of Chil-TAMs and CqTAMs in a spontaneous mouse model of pancreatic cancer. We used the iKras* and iKras* p53* genetically engineered mouse models of pancreatic cancer that express oncogenic Kras ${ }^{G 12 D}$ in the pancreas epithelium in an inducible and reversible manner (Collins et al, 2012a, 2012b). The iKras* mice represent an early lesion timepoint, whereas the iKras* p53* mice, which have a pancreas specific mutated p53, represent a late lesion timepoint, allowing us to evaluate Chil-TAMs and Cq-TAMs during progression of PDA. We first subcutaneously implanted scaffolds into control and iKras* p53* mice that had oncogenic Kras expression for 15 wk. Oncogenic Kras continued to be expressed for the duration of the experiment. We harvested the scaffolds 3 wk later and performed single-cell RNA sequencing on the scaffold infiltrate (Figs 5A and S5A and B). We observed an increase in C1qa, C1qb, and Chil3, but not Trem2, in the

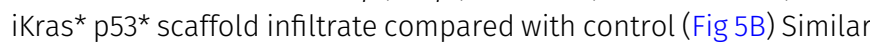
to the orthotopic scaffolds, we observed two distinct macrophage populations (Fig 5C). SAM 1 was defined by expression of C1qa, C1qb, and Trem2, whereas SAM 2 was defined by expression of Chil3, Plac8, and Ly6C2 (Fig 5C and D). 

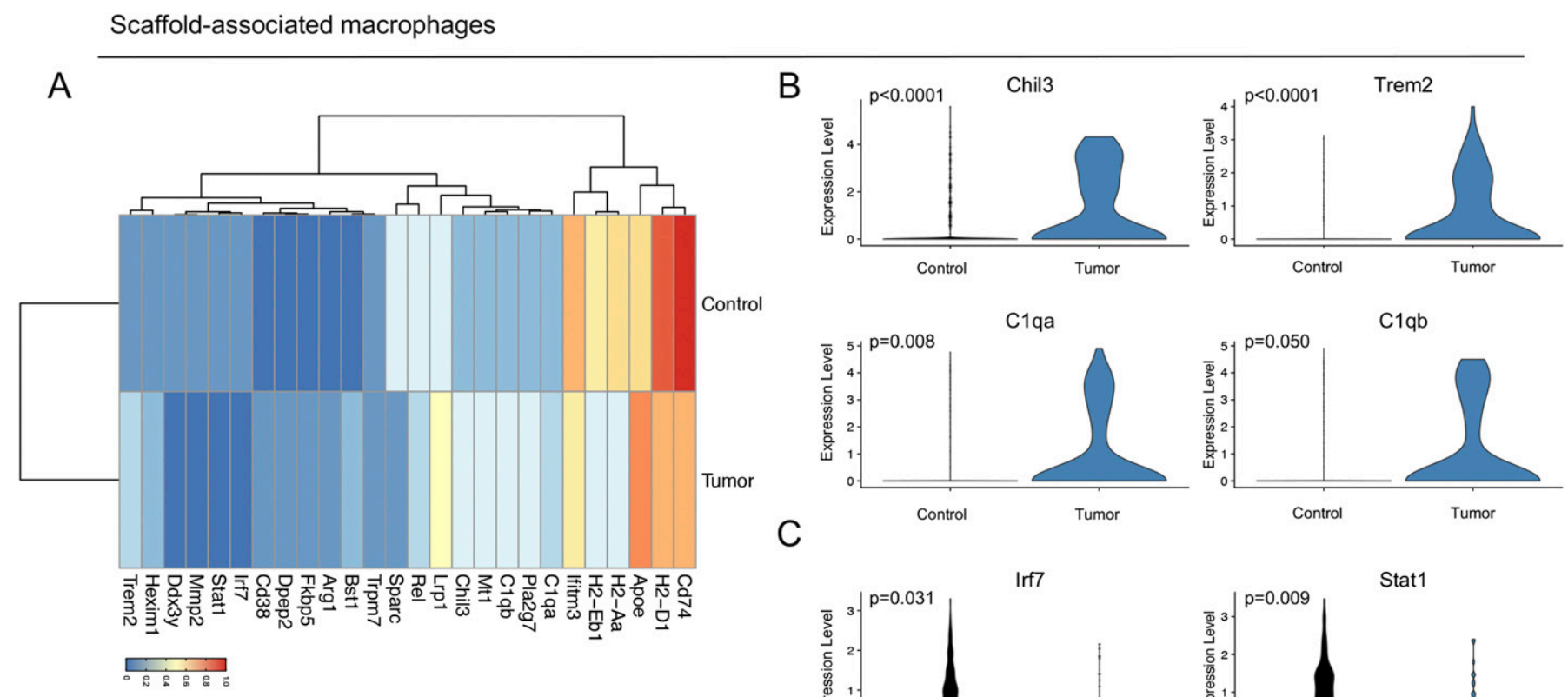

C


Scaffold-associated macrophages


$\mathrm{F}$
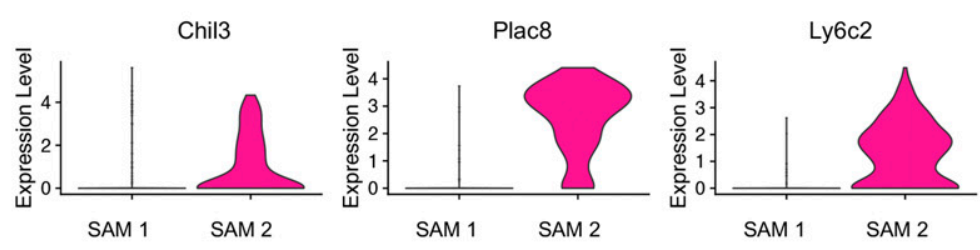

Figure 3. Identification of two distinct macrophage subsets in scaffold infiltrate.

(A) Average expression heat map for select differentially expressed genes between macrophages from control and tumor-bearing (TB) scaffolds. Low expression is shown in blue and high expression in red. All genes plotted are statistically significant, determined using nonparametric Wilcoxon rank sum test with a $P$-value cut off of $P<0.05$. (B, C) Violin plot of normalized gene expression of select up-regulated and (C) down-regulated genes in macrophages from control (black) and TB (blue) scaffolds. Statistically significant genes were determined using non-parametric Wilcoxon rank sum test with a $P$-value cutoff of $P<0.05$. (D) UMAP visualization of scaffold-associated macrophage (SAM) 1 (navy) and SAM 2 (pink) subsets in control and

TB scaffolds. (E, F) Violin plots of normalized expression of C1qa, C1qb, and Trem2 in SAM 1 and (F) Chil3, Plac8, and Ly6c2 in SAM 2.

By single-cell RNA sequencing, we compared the prevalence of Chil-TAMs and Cq-TAMs in iKras* and iKras* p53* pancreas samples compared with the normal pancreas (Figs S5A, C, and D). The macrophages unbiasedly clustered into three distinct populations (Fig 5E) including Chil-TAMs and Cq-TAMs (Fig 5E and F). We additionally identified a third population of macrophages defined by high expression of $\mathrm{C} C \mathrm{r} 2$, and $\mathrm{Cd} 74$ and $\mathrm{H} 2$-Eb1, the latter encoding components of the MHC complex (Fig S5E). We then compared iKras* samples and iKras* $553^{*}$ samples, reflecting early and late stages of PDA progression, and observed increased expression in Chil-TAM and
Cq-TAM makers in the infiltrating macrophages, along with a loss of CCR2-TAM markers in advanced lesions (Figs 5G and S5F). Thus, over time, the specific macrophage signature becomes more pronounced.

\section{Macrophages in human pancreatic cancer overexpress TREM2 and complement genes}

Because there is no human ortholog for Chil3/Ym1 (Kzhyshkowska et al, 2007) or Ly6c2 (Lee et al, 2013), we focused on PLAC8, TREM2, and the complement components C1QA and C1QB to analyze human 
Orthotopic KPC tumor single cell RNA sequencing
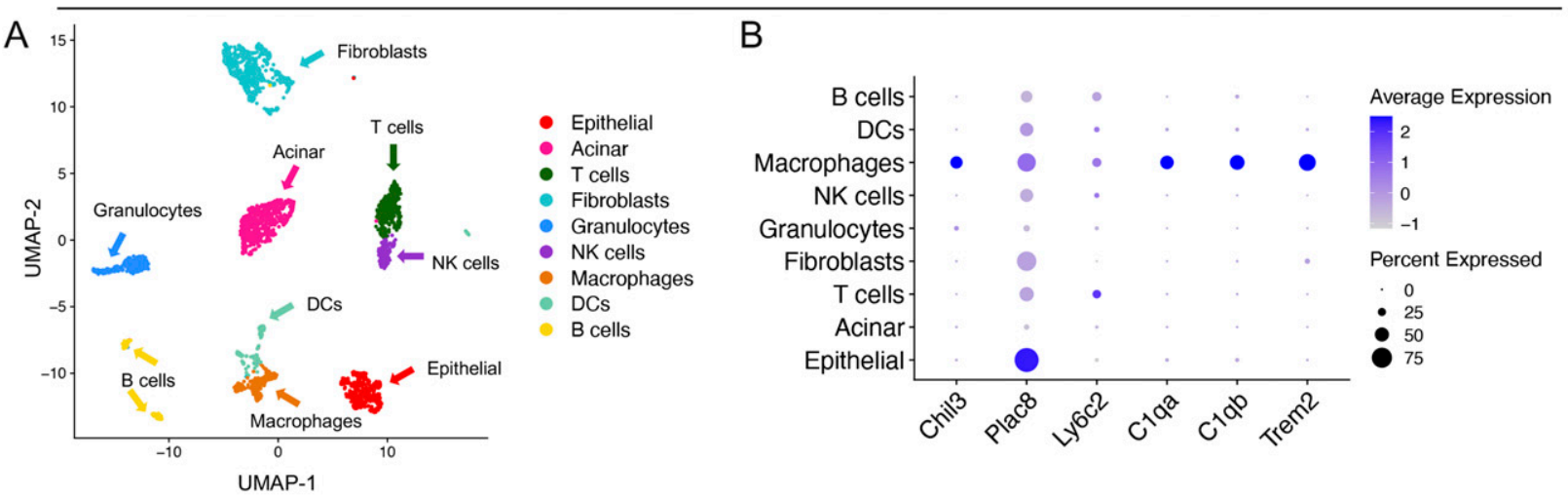

Orthotopic KPC TAMs
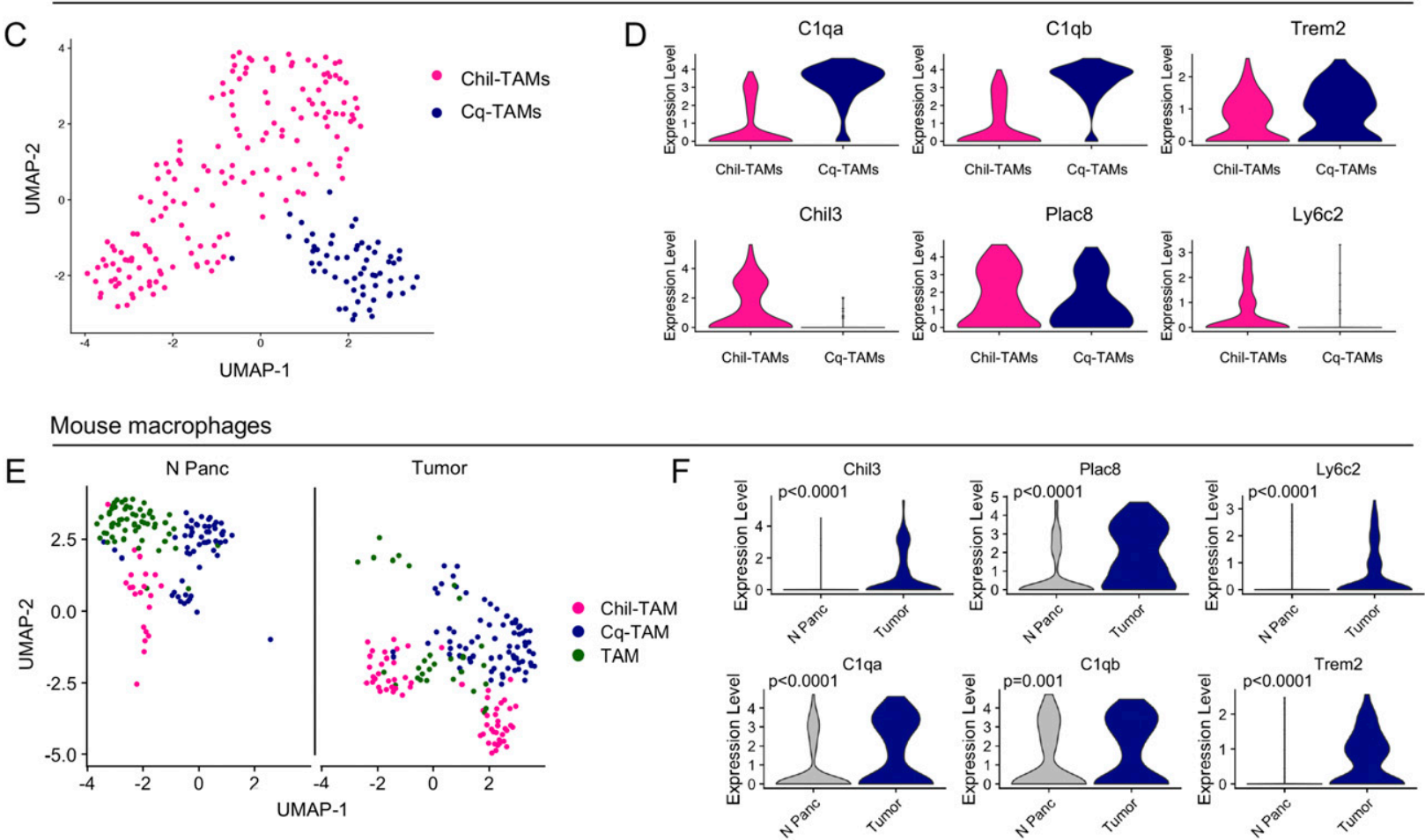

G
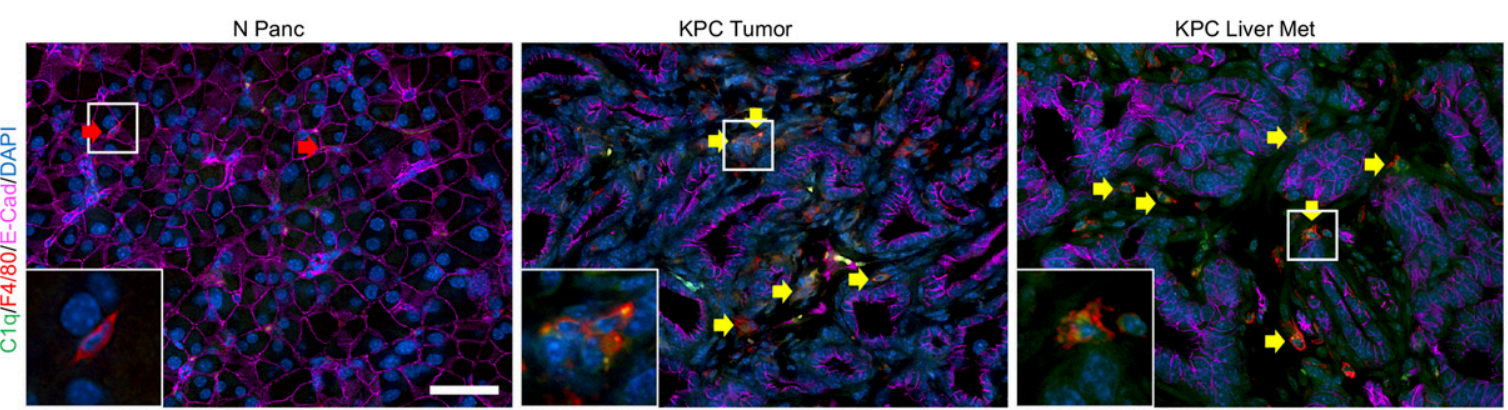

Figure 4. Macrophages in mouse pancreatic tumors overexpress TREM2 and complement genes.

(A) UMAP visualization of mouse orthotopic pancreatic tumors $(n=2)$. (B) Dot Plot of scaffold-associated macrophage signature, Chil3, Plac8, Ly6c2, C1qa, C1qb, and Trem2 in identified cell populations in the orthotopic KPC tumors. Color represents average expression, whereas size of dot represents percent expressed. (C) UMAP visualization of Chil-tumor-associated macrophages (TAMs) (pink) and Cq-TAMs (navy) subsets in mouse orthotopic pancreatic tumors. (D) Violin plots of C1qa, C1ab, Trem2, Chil3, Plac8, and Ly6c2 across Chil-TAMs and Cq-TAMs. (E) UMAP visualization of Chil-TAM (pink), Cq-TAM (navy), and TAM (green) macrophage subsets in normal pancreas $(n=1)$ and orthotopic tumors $(n=2)$. $(F)$ Violin plot of normalized gene expression of Chil3, Plac8, Ly6c2, C1qa, C1qb, and Trem2 in macrophages from normal 
patient samples. To assess the expression of TREM2, C1QA, and $C 1 Q B$, and PLAC8 we queried a single-cell RNA sequencing dataset including human normal/adjacent normal pancreas $(n=3)$ and human PDA tumors $(n=16)$ (Steele et al, 2020) (Fig 6A). Consistent with our observation in mice, TREM2, C1QA, and C1QB were mainly expressed in macrophages, whereas PLAC8 was expressed in multiple cell types, including macrophages (Fig 6B). The macrophages separated into two transcriptionally distinct subsets, which were consistent across patients (Figs $6 \mathrm{C}$ and S6A and B). One population was enriched for expression of C1QA, C1QB, and TREM2 (CQ-TAMs), whereas the other population had higher expression of PLAC8, VCAN, FABP5, and RETN (TAMS) (Figs 6D and S6A and C). Paralleling the mouse data, C1QA, C1QB, and TREM2 were upregulated in macrophages from human pancreatic cancer compared with macrophages from the non-malignant pancreas (Fig 6E).

\section{Macrophages in human liver metastases express high levels of TREM2 and complement genes}

To further address the role of CQ-TAMs in the systemic immune response, we next assessed the expression of the macrophage signature genes in liver metastasis samples from PDA patients $(n=5)$. These samples were obtained through ultrasound guided percutaneous biopsy of a liver lesion in five individual PDA patients and processed for single-cell RNA sequencing. Single-cell RNA sequencing followed by UMAP visualization revealed a profound stromal response, including a substantial population of macrophages within the metastatic liver lesions (Figs 7A and S7A). Similar to our scaffold and primary tumor data, the macrophages in the liver metastases had high expression of C1QA, C1QB, and TREM2 consistent with this macrophage population being part of a systemic response to a primary tumor (Fig 7B). In addition, subsetting of the liver metastasis associated-macrophages confirmed the existence of two transcriptionally distinct macrophage populations (i.e., CQ-TAMs and TAMs), similar to the findings in the scaffolds in mice and primary tumors in mice and humans (Fig 7C). The signature genes C1QA, C1QB, and TREM2 had highest expression in CQ-TAMs compared with TAMS (Figs 7D and S7B). CQ-TAMs are present at both the primary tumor and systemic locations in humans. Similar to our analysis in mice, we next performed differential expression analysis on macrophages from human liver metastases compared with macrophages from human primary tumors (Fig 7E and F). IL1A was enriched in both scaffolds and liver metastases compared with the primary tumor (Figs 7F and S4F). ILIA has been associated with increased cell invasion in vitro in PDA (Melisi et al, 2009).

\section{Complement-high myeloid cells are elevated in the blood of pancreatic cancer patients}

The notion that systemic changes in the immune/myeloid gene expression signature might reflect the presence of a primary tumor is potentially important to add to the diagnostic/prognostic toolbox. With this in mind, we assessed the macrophage gene expression signature in human blood. We used a published dataset of single-cell RNA sequencing on PBMCs from healthy donors $(n=4)$ and PDA patients $(n=16)$ (Steele et al, 2020) and queried it for the expression of our signature genes: C1QA, C1QB, and TREM2 (Fig S8A). We observed highest expression of C1QA, C1QB, and TREM2 in circulating monocytes in human PBMCs (Fig 8A). We identified four populations of circulating monocytes based on expression of CD14 and CD16 (FCGR3A/B) as previously defined (Wong et al, 2011) (Figs $8 \mathrm{~B}$ and $\mathrm{S} 8 \mathrm{~B}$ ). Similar to the scaffold, liver and primary tumor, C1QA, $C 1 Q B$, and TREM2 marked only one subpopulation of monocytes (CQ-Monocytes) in human PBMCs (Figs 8 C and S8B-D). Interestingly, PLAC8 was highest in Monocyte populations 2 and 3, suggesting that it marks distinct populations from the CQ-monocytes (Figs $8 \mathrm{C}$ and S8D). To assess whether these genes are up-regulated in the blood of PDA patients we further compared PBMCs between healthy donors and PDA patients and saw higher normalized expression of $C 1 Q A$ and $C 1 Q B$ in patients, suggesting that the up-regulation of these markers also applies to circulating monocytes (Figs 8D and S8E).

In summary, we have identified a complement-high population of macrophages, CQ-TAMs, which exists both at the primary tumor and systemically in mouse and human pancreatic cancer. CQ-TAM marker expression is enriched at the primary tumor and in circulation in human PDA patients, presenting a novel population of monocytes/macrophages that could potentially serve as indicators of disease state.

\section{Discussion}

In this study, we used bioengineered scaffolds as a tool to discover a novel gene signature that is associated with tumor-bearing mice, including elevated expression of C1qa, C1qb, and Trem2. By singlecell RNA sequencing we mapped this signature to a population of SAMs and determined that a corresponding TAM population ( $\mathrm{Cq}-$ TAMs) is present at the primary tumor in multiple mouse models of PDA. We then analyzed single-cell RNA sequencing data from patient tumors (Steele et al, 2020) and novel single-cell RNA sequencing data from liver metastases and identified macrophages expressing high levels of $C 1 Q A, C 1 Q B$, and TREM2 in both primary tumor and metastases. Finally, we determined that $C 1 Q A$ and $C 1 Q B$ expression is enriched in pancreatic cancer patient blood compared with healthy individuals, suggesting that the elevation of these markers may serve as a novel predictor of disease in PDA patients.

Biomaterial scaffolds model the pre-metastatic niche (Azarin et al, 2015; Rao et al, 2016; Bushnell et al, 2019) and allow for repeated sampling, and, thus, longitudinal analyses. Furthermore, scaffolds model natural secondary sites of metastasis and are distinct from

pancreas (grey) and orthotopic tumors (navy). Statistically significant genes were determined using non-parametric Wilcoxon rank sum test with a $P$-value cut offof $P<$ 0.05. (G) Co-immunofluorescence of normal mouse pancreas (N Panc), KPC tumor, and KPC liver metastasis samples of C1q (green), F4/80 (red), E-Cadherin (pink) and DAPI (blue). Red arrow denotes $\mathrm{Clq}^{-} \mathrm{F} 4 / 80^{+}$macrophage in the normal pancreas. Yellow arrows denote $\mathrm{C} \mathrm{q}^{+} \mathrm{F} 4 / 80^{+}$macrophages in $\mathrm{KPC}$ tumor and $\mathrm{KPC}$ liver metastasis. Inlets show higher magnification of select macrophages in boxed region. Scale bars, $50 \mu \mathrm{m}$. 

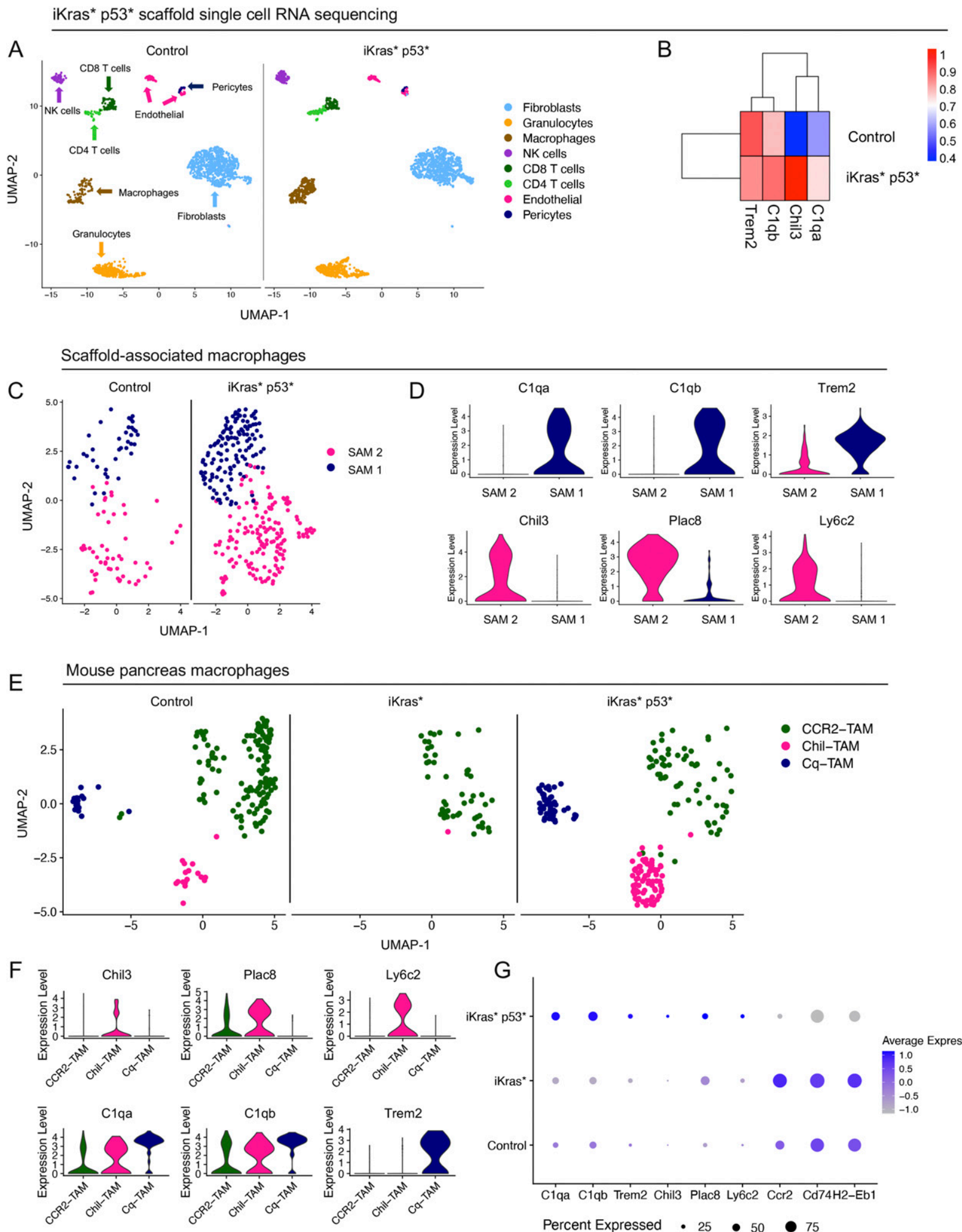

Figure 5. Cq-tumor-associated macrophage (TAM) and Chil-TAM markers are elevated in the iKras* $\mathrm{p} 53^{*}$ model of pancreatic cancer.

(A) UMAP visualization of scaffolds from control $(n=1)$ and iKras* p53* mice $(n=1)$. (B) Average expression heat map of Trem2, C1qb, Chil3, and C1qa in control and iKras* p53* scaffolds. High expression is in red, whereas low expression is in blue. (C) UMAP visualization of scaffold-associated macrophage (SAM) 1 (navy) and SAM 2 (pink) macrophage subsets in control and iKras* p53* scaffolds. (D) Violin plots of C1qa, C1qb, Trem2, Chil3, Plac8, and Ly6c2 across SAM 1 and SAM 2. (E) UMAP visualization of CCR2-TAM (green), Chil-TAM (pink), and Cq-TAM (navy) macrophage subsets in control, iKras* and iKras* p53* pancreas samples. (F) Violin plots of Chil3, Plac8, Ly6c2 C1qa, C1qb, and Trem2 across CCR2-TAM, Chil-TAM, and Cq-TAM macrophage subsets. (G) Dot plot of C1qa, C1qb, Trem2, Chil3, Plac8, Ly6c2, CCr2, Cd74, and H2-Eb1 in Control, iKras* and iKras* p53* macrophages. Color represents average expression. Size of the dot represents percent expressed. 

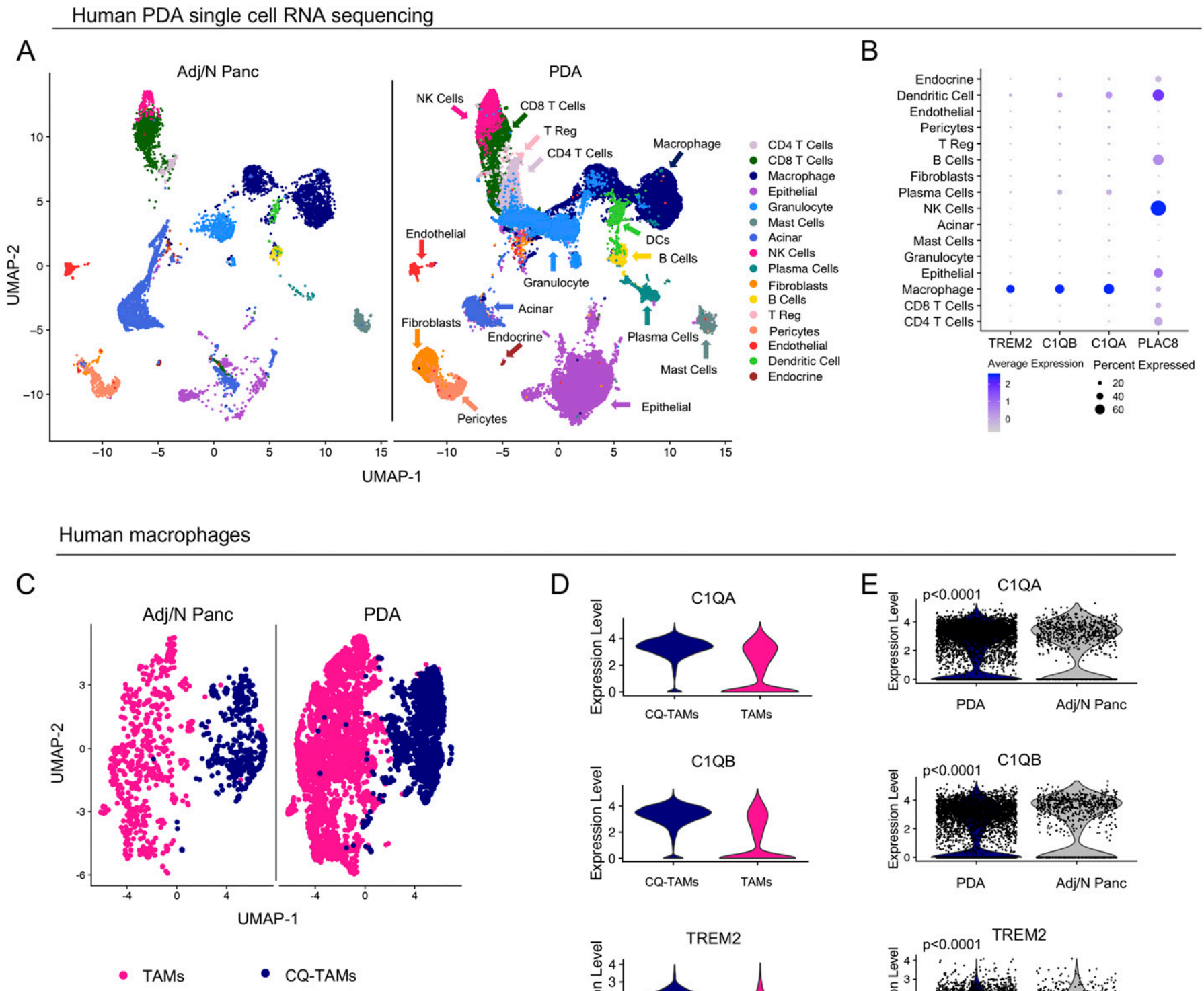
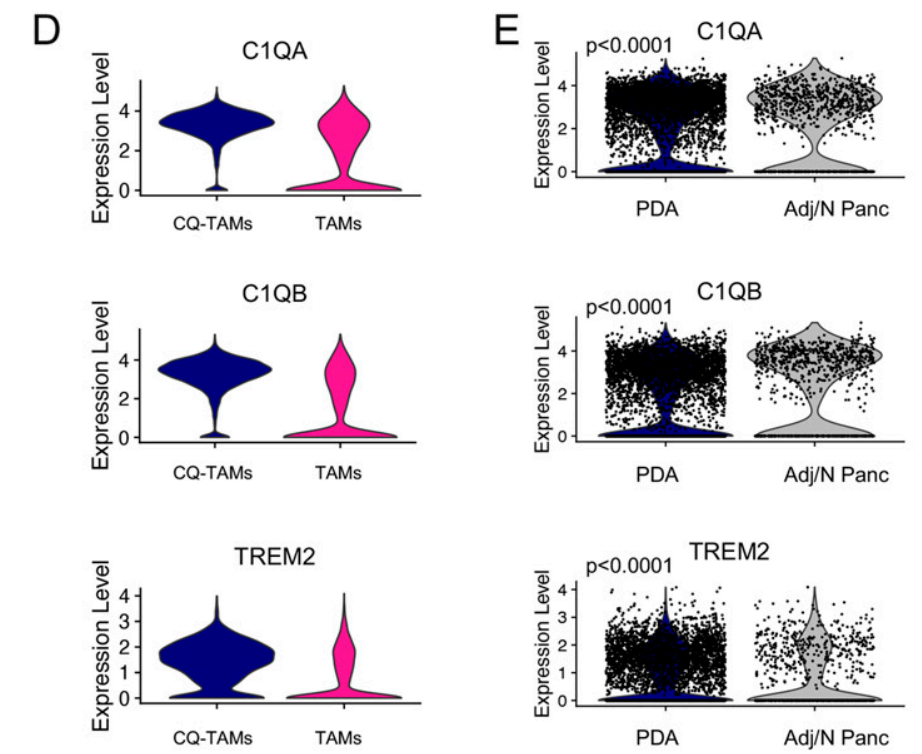

Figure 6. Macrophages in human pancreatic tumors overexpress TREM2 and complement genes.

(A) UMAP visualization of Adj/Norm $(n=3)$ and pancreatic ductal adenocarcinoma (PDA) tumors ( $\mathrm{n}=16)$. (B) Dot plot of TREM2, C1QB, C1QA, and PLAC8 in human PDA tumor cell populations. Color of the dot represents average expression, whereas the size of the dot represents expression frequency. (C) UMAP visualization of human tumor-associated macrophages (TAMs) (pink) and CQ-TAMs (navy) from adjacent normal pancreas ( $n=3$ ) and human PDA tumors ( $n=16$ ). (D) Violin plots of C1QA, C1QB, and TREM2 in human TAMs and CQ-TAMS. (E) Violin plots of C1QA, C1QB, and TREM2 in human macrophages from human PDA tumors compared with adjacent normal pancreas. Statistics were determined using non-parametric Wilcoxon rank sum test with a $P$-value of $P<0.0001$.

blood, as recently determined (Oakes et al, 2020; Morris et al, 2020a). Relevant to our study, myeloid cells entering scaffolds differentiate into macrophages, distinct from peripheral blood monocytes.

PDA is characterized by a dense, fibroinflammatory stroma, which contains a large infiltration of immunosuppressive myeloid cells. Myeloid cells are a heterogeneous population consisting of MDSCS and TAMs that contribute to tumor progression and metastasis (Qian \& Pollard, 2010). Although TAMs have been welldescribed as contributors to PDA tumor progression, no prior study has examined their role systemically in response to a primary tumor. Here, we have leveraged single-cell RNA sequencing analysis to identify two distinct systemically induced macrophage populations that are specific to mouse and human pancreatic cancer. In mice one macrophage population upregulated Chil3 (Chil-TAMs) in response to disease, whereas the other population up-regulated C1qa, C1qb, and Trem2 (CqTAMs) in mouse and PDA patients. The role for these genes is unknown in pancreatic cancer. 


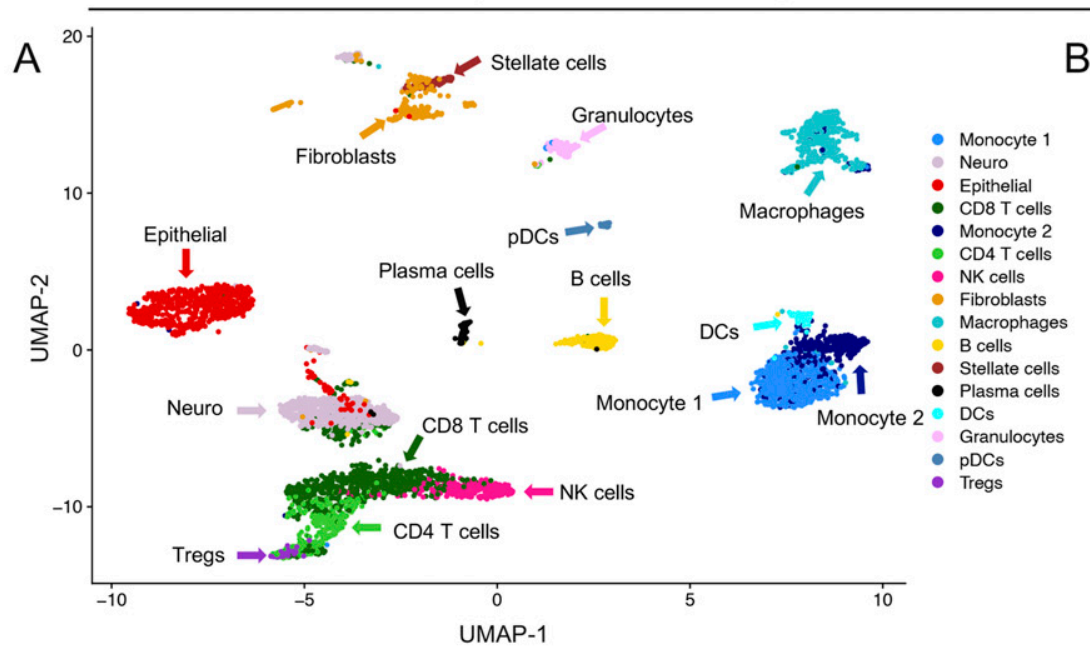

B

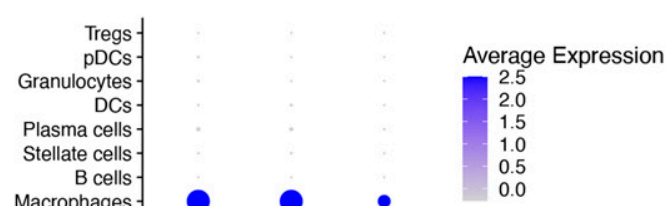

Macrophages
Fibroblasts

Fibroblasts

NK cells

Monocyte 2

CD8 T cells

Epithelial

Neuro

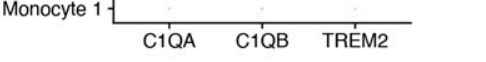

Percent Expressed

- 0

- 20

- 40

60
80

UMAP-1

Human liver metastases macrophages
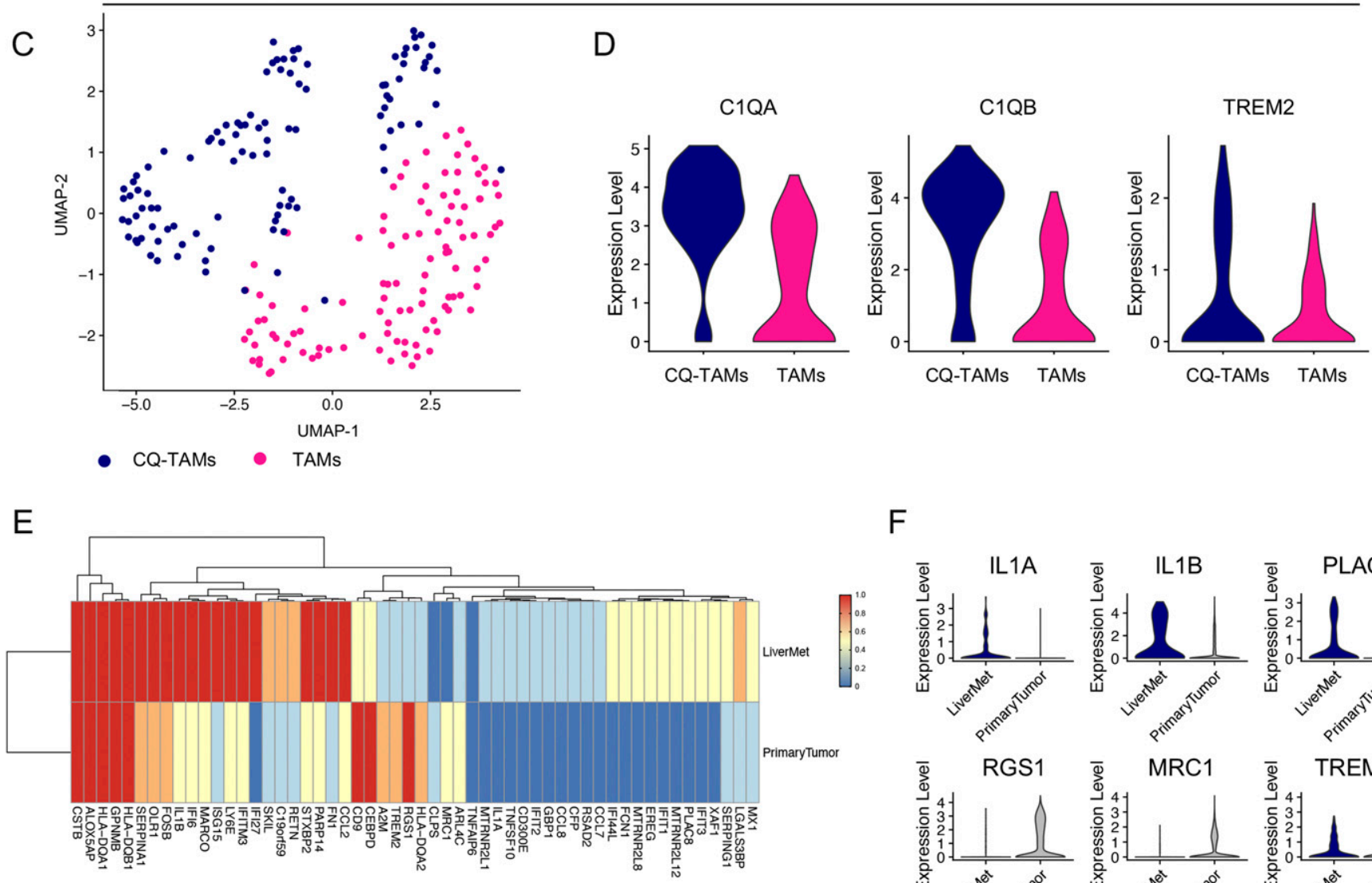

$\mathrm{F}$

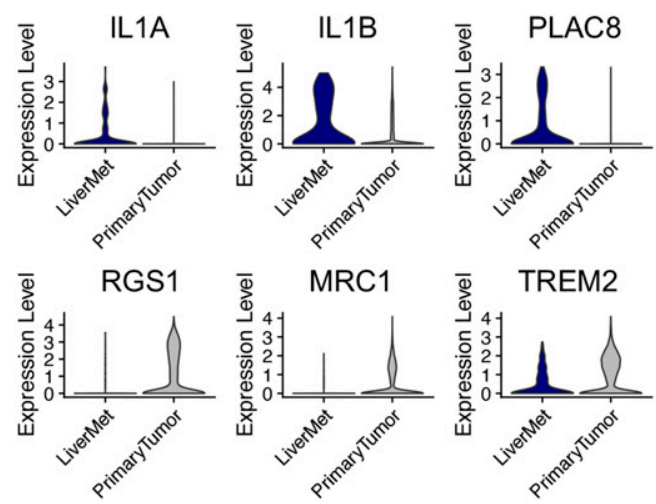

Figure 7. Macrophages in human liver metastases express high levels of TREM2 and complement genes.

(A) UMAP visualization of human liver metastasis samples $(n=5)$ from pancreatic ductal adenocarcinoma patients. (B) Violin plots of normalized expression of C1QA, $C 1 Q B$, and TREM2 in identified cell populations in the liver metastasis lesions from human pancreatic ductal adenocarcinoma patients $(n=5)$. (C) UMAP visualization of CQ-tumor-associated macrophages (TAMs) (navy) and TAMs (pink) identified in human liver metastasis samples. (D) Violin plots of normalized expression for C1QA, C1QB, and TREM2 in CQ-TAMs and TAMs from liver metastasis samples. (E) Average expression heat map for select differentially expressed genes between macrophages from human liver metastases and human primary tumors. Low expression is shown in blue and high expression in red. All genes plotted are statistically significant, determined using nonparametric Wilcoxon rank sum test with a P-value cutoff of $P<0.05$. (F) Violin plots of normalized expression for IL1A, IL1B, PLAC8, RGS1, MRC1, and TREM2 in macrophages from human liver metastasis and primary tumor samples. 
A

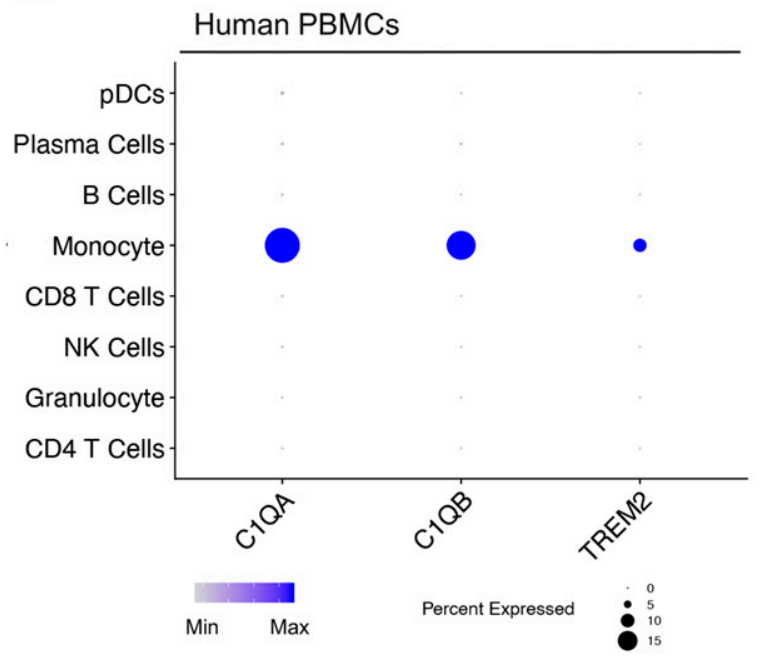

B

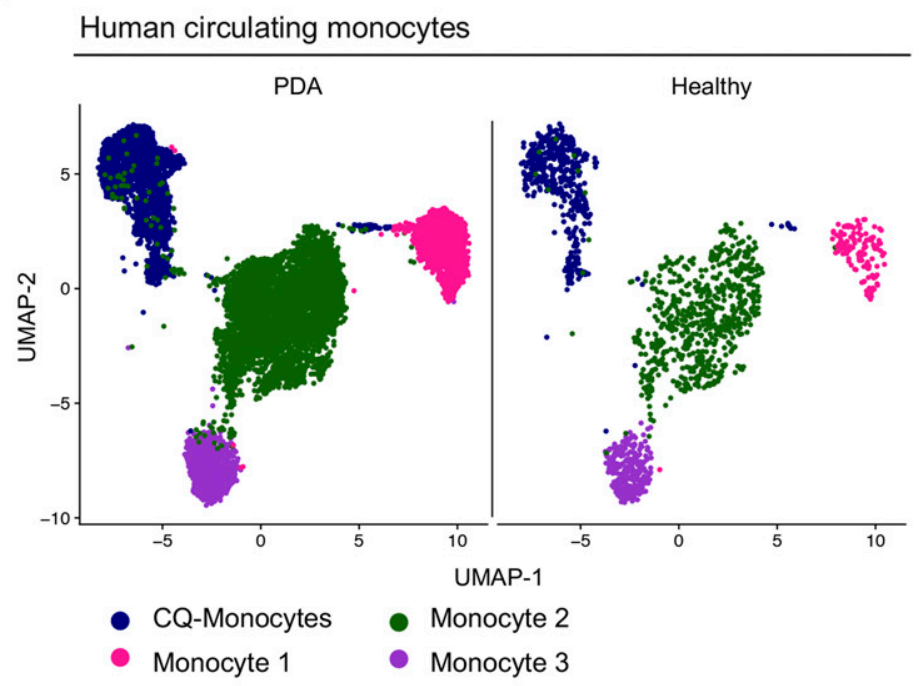

Human circulating monocytes

C
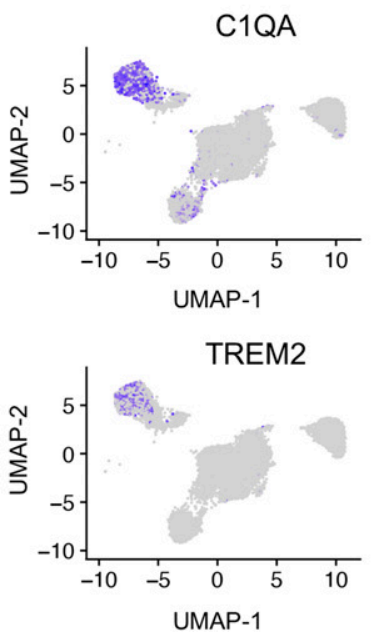
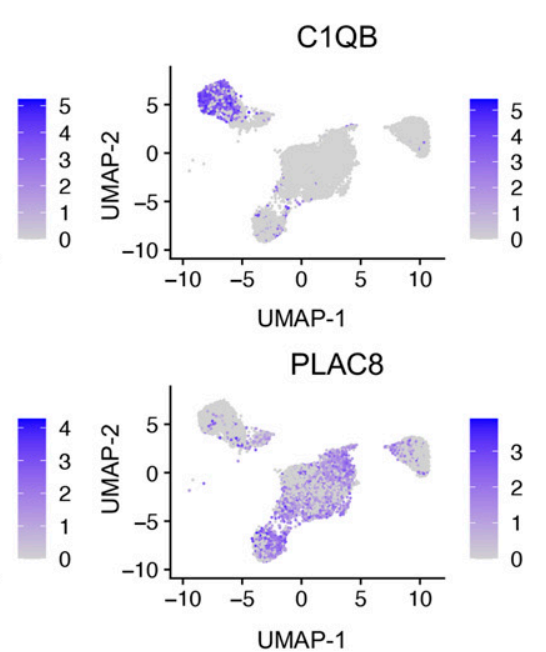

PLAC8

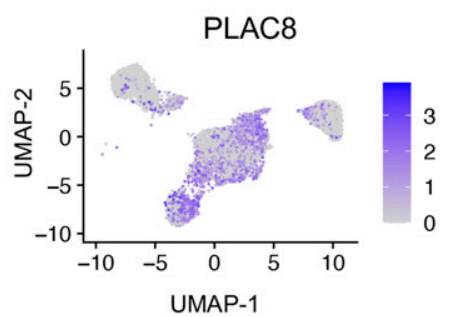

$\mathrm{D}$

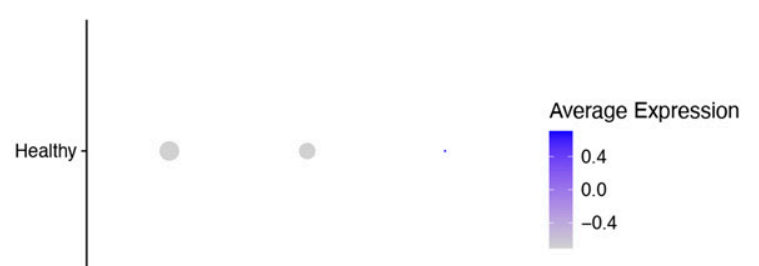

Percent Expressed

1.0

- 1.5

2.0
25

Figure 8. Complement-high monocyte markers are elevated in the blood of pancreatic cancer patients.

(A) Dot plot of C1QA, C1QB, and TREM2 in identified populations in human PBMCs. Color of the dot represents average expression, whereas the size of the dot represents expression frequency. (B) UMAP visualization of CQ-monocytes (navy), monocyte 1 (pink), monocyte 2 (green), and monocyte 3 (purple) in human PBMCs in pancreatic ductal adenocarcinoma $(n=16)$ and healthy $(n=4)$. (C) Feature plot of C1QA, C1QB, TREM2, and PLAC8 in human monocyte subsets in the blood. Blue is high expression and grey is low expression. (D) Dot plot of C1QA, C1QB, and TREM2 in PBMCs from healthy donors and pancreatic ductal adenocarcinoma patients. High expression is in blue, low expression is in grey. Size of the dot represents expression frequency.

C1QA and C1QB are components of the complement cascade. The complement cascade is a crucial mediator of innate immunity and can be recruited by components of the adaptive immune system to combat microbial infection, but recently its role in cancer and the tumor microenvironment has been explored (Bonavita et al, 2015; Afshar-Kharghan, 2017). Up-regulation of C1QB has been reported in PBMCs of melanoma patients (Luo et al, 2011). Although C1QA and C1QB have not been extensively studied, a recent report examined the role of the complement cascade in PDA. Zhang et al (2019) reported that TAMs help tumor cells avoid complement-mediated cell death, providing mechanistic insight into TAM and complement component cross-talk in pancreatic cancer (Zhang et al, 2019). Our data provide evidence for up-regulation of the complement components C1QA and C1QB in PDA TAMs systemically. Further work is needed to determine if the up-regulation of complement components is a side effect of the systemic inflammation caused by PDA or if it is functionally contributing to carcinogenesis (Bettac et al, 2017).

To our knowledge, TREM2 has not been evaluated in pancreatic cancer, but has been shown to play an immunosuppressive role in other tumor types (Katzenelenbogen et al, 2020; Molgora et al, 2020). Its family member, triggering receptor expressed on myeloid cells 1 (TREM1), however, has been implicated to reduce tumor burden in PDA (Shen \& Sigalov, 2017). Whereas understudied in PDA, 
TREM2 has been extensively evaluated in Alzheimer's disease, a neurodegenerative disease, which, like cancer, is marked by a chronic inflammatory response (Kinney et al, 2018). TREM2 is a risk factor for Alzheimer's disease and is believed to modulate the behavior of microglia to exacerbate the inflammatory response.

A similar single-cell sequencing approach to ours previously identified two distinct macrophage subsets in normal renal tissue across multiple species (Zimmerman et al, 2019). The authors reported a population of inflammatory macrophages defined by high expression of Ly6c, Plac8, and Chil3 and a resident macrophage subset defined by high expression of Cd81, C1qa, C1qb, and C1qc. Given the similarity of their finding to ours, these macrophage populations are likely relevant in other model systems. The gene signature presented here identified markers that define macrophage/monocyte subsets in mouse and human pancreatic cancer. The identification of a tumor associated signature in blood monocytes will potentially be exploited for diagnostic and prognostic applications in pancreatic cancer patients.

\section{Materials and Methods}

\section{Study approvals}

All animal procedures and studies were performed at the University of Michigan (Protocol Number PRO00007983) in compliance with the Institutional Animal Care \& Use Committee (IACUC) guidelines. For human research, this study included a dataset that included patients over the age of $18 \mathrm{yr}$ who received diagnostic endoscopic ultrasound for a suspected pancreas mass who were consented under the Institutional Review Board HUM00041280 (Two additional passes using a 22 Gauge SharkCore needle was performed for research once biopsy for clinical use was obtained). For surgically resected tissue, patients who underwent either Whipple of distal pancreatectomy were consented under Institutional Review Board HUM00025339. For PBMC collection, up to $40 \mathrm{ml}$ of whole blood was collected pre- and intraoperatively for all consented patients. All patients provided written consent and procedures and studies performed were carried out in accordance to ethical standards. For liver metastasis samples, patients over the age of 18 referred for percutaneous liver biopsy of a mass suspected to be metastatic PDA were consented according to HUM00025339. Up to 2 extra biopsies were taken for research.

\section{Scaffold fabrication}

Implantable, biomaterial scaffolds were formed by mixing polycaprolactone microspheres with $\mathrm{NaCl}$ particles $(250-425 \mu \mathrm{m})$ at a 1:30 (w/w) ratio as previously described (Rao et al, 2016). This mixture was then pressed into a $5 \mathrm{~mm}$ (diameter) by $2 \mathrm{~mm}$ (height) disc, heated at $60^{\circ} \mathrm{C}$ for 5 min on each side, and submerged in water to remove salt particles, leaving a porous structure. The scaffolds were then sterilized in $70 \%$ ethanol and stored in $-80^{\circ} \mathrm{C}$ until surgical implantation.

\section{Animal experiments}

\section{Mice}

C57/BL6J mice (stock number \#000664; Jackson Laboratory), KPC (Hingorani et al, 2005), iKras* (Collins et al, 2012a), and iKras* p53* (Collins et al, 2012b) mice were used for mouse experiments. All mice were housed in the Rogel Cancer Center vivarium at the University of Michigan. Experimental mice were monitored daily.

\section{Doxycycline treatment}

iKras* and iKras* p53*mice were administered doxycycline chow at 8 wk of age (F3949; BioServ) to induce expression of $\mathrm{Kras}^{\mathrm{G12D}}$ for $72 \mathrm{~h}$, followed by $2 \mathrm{~d}$ of eight intraperitoneal injections of caerulein (75 $\mu \mathrm{g} / \mathrm{kg}$; Sigma-Aldrich) to induce pancreatitis, as previously described (Collins et al, 2012a). Control mice lacked the full set of alleles and were administered doxycycline chow and caerulein along with experimental animals. For early lesion samples, iKras* mice had continuous doxycycline administration for 3 wk after caerulein. For tumor samples, iKras* p53* mice were continuously administered doxycycline for $14 \mathrm{wk}$. Scaffolds were subcutaneously

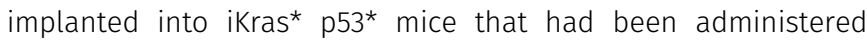
doxycycline for $15 \mathrm{wk}$ and harvested 3 wk later.

\section{Scaffold implantation}

Mice were anesthetized using isoflurane and the surgical area was prepared using aseptic technique. Before implantation, scaffolds were warmed at RT for $30 \mathrm{~s}$ and then implanted subcutaneously in C57/BL6J or iKras* p53* mice. The incision site was closed using absorbable sutures (\#)303H; Ethicon). For all experiments, up to eight scaffolds were implanted per mouse to allow enough cells for downstream analysis. For orthotopic tumor studies, 7940b (BL/6) cells derived from the $L S L-K r a S^{G 12 D /+} ;$ LSL-Trp53 ${ }^{R 172 H /+} ; P d \times 1-C r e$ (KPC) model of pancreatic cancer (A gift from Dr. Gregory Beatty, University of Pennsylvania) were orthotopically transplanted into the pancreas $1 \mathrm{wk}$ after scaffold implantation.

\section{Orthotopic transplantation model}

Orthotopic transplantation into the pancreas was performed as previously described (Aiello et al, 2016). Briefly, $5 \times 10^{4} 7940 \mathrm{~b} \mathrm{KPC}$ $(\mathrm{BL} / 6)$ cells were prepared in a 1:1 ratio of growth-factor reduced Matrigel and media (DMEM supplemented with 10\% FBS). Mice were anesthetized using isoflurane and the surgical area was prepared using aseptic technique. A tumor cell suspension of $50 \mu \mathrm{l}$ was injected directly into the pancreas using an insulin syringe. Control, non-tumor-bearing mice in scaffold experiments received injection of $50 \mu \mathrm{l}$ of $50 \%$ Matrigel in media.

\section{Histopathological analysis}

Scaffolds were removed from $-80^{\circ} \mathrm{C}$ and stored on dry ice until embedding. For frozen sections, scaffolds were embedded in optimal cutting temperature and allowed to solidify over dry ice, then stored at $-80^{\circ} \mathrm{C}$ until sectioning. Frozen sections were cut at $10 \mu \mathrm{m}$. For immunofluorescent staining on optimal cutting temperature embedded scaffolds, slides were brought to RT and then submerged in 4\% PFA for 12 min at RT, and then washed with three 
changes of PBS. Scaffolds were then blocked with 1\% BSA in PBS for $1 \mathrm{~h}$ at RT, followed by primary antibody incubation overnight at $4^{\circ} \mathrm{C}$ and secondary antibody incubation for $45 \mathrm{~min}$ at RT. Cell nuclei were counterstained with Prolong Diamond Antifade Mountant with DAPI (Invitrogen). Tissues were fixed overnight in 10\% buffered formalin, then transferred to $70 \%$ ethanol for paraffin embedding. Immunohistochemical staining was performed on tissue sections using the Ventana Discovery Ultra XT autostainer and counterstained with hematoxylin. Scaffolds and tissues were imaged on the Olympus BX53F microscope with the Olympus DP80 digital camera and CellSens Standard software using the $20 \times$ and $40 \times$ objectives. Quantitation of positive immunohistochemical stain was performed using Image J, Fiji V2.0.0-rc-69/1.52p on at least three 20x magnification fields per sample. For co-immunofluorescence, Alexa Fluor 488 Tyramide SuperBoost Kit (Invitrogen) with SignalStain EDTA Unmasking Solution (Cell Signaling) were used for C1q staining according to manufacturer's protocols, then Alexa Fluor (Invitrogen) secondary antibodies were used for F4/80 and E-cad. Cell nuclei were counterstained with Prolong Diamond Antifade Mountant with DAPI (Invitrogen). Images were taken using Olympus BX53F microscope, Olympus DP80 digital camera, and CellSens Standard software. A list of the antibodies used and corresponding dilutions can be found in Table S3.

\section{Mass cytometry (СуTOF)}

To obtain a single-cell suspension, scaffolds were first enzymatically digested with $1 \mathrm{mg} / \mathrm{ml}$ Collagenase $\mathrm{P}$ in DMEM for $10 \mathrm{~min}$ at $37^{\circ} \mathrm{C}$ under constant agitation. Scaffolds were then mechanically digested and allowed further enzymatic digestion for an additional $10 \mathrm{~min}$. Cells are then filtered through a $40-\mu \mathrm{M}$ mesh. Preparation of the mouse tissue for CyTOF was performed as previously described (Zhang et al, 2020). Mouse livers were mechanically and enzymatically digested for 10 min at $37^{\circ} \mathrm{C}$ under agitation and filtered through a 40- $\mu \mathrm{M}$ mesh to obtain single cells. For mouse PBMCs, up to $1 \mathrm{ml}$ of whole blood was obtained via cardiac puncture into EDTAcoated syringes and transferred to $1.5-\mathrm{ml}$ tubes. Tubes were inverted 10 times and centrifuged at RT at 1,700 $\mathrm{g}$ for $20 \mathrm{~min}$. Serum was then removed and the PBMC layer was transferred to a new tube. PBMCs were washed, underwent ammonium-chloridepotassium (ACK) lysis for $10 \mathrm{~min}$ at RT, and were then centrifuged at $300 \mathrm{~g}$ for $5 \mathrm{~min}$. For both scaffolds, PBMCs and tissues, up to $1 \times 10^{7}$ cells from the single-cell suspension were stained with the live/dead marker, Cell-ID Cisplatin (\#201064; Fluidigm) for 5 min at RT. Maxpar cell surface staining protocol was followed (PN 400276 A4). Cells were stained with a panel of surface antibodies (additional details can be found in Table S1) for 30 min at RT and then stored in Cell-ID Intercalator-IR (201192A; Fluidigm) until being shipped and acquired on the CyTOF2 Mass Cytometer at the University of Rochester Medical Center. Downstream analysis on normalized FCS files was performed using the Premium CytoBank Software V7.3.0 (cytobank.org).

\section{Inflammatory gene array and signature}

Scaffolds were removed from the subcutaneous space and flash frozen in liquid nitrogen, then stored at $-80^{\circ} \mathrm{C}$. Scaffolds were submerged in TRI Reagent (\#R2050-1-50) and mechanically homogenized. RNA was extracted using Direct-zol RNA miniprep (\#R2051) with on column DNase I treatment. RNA quality was determined using both NanoDrop results for concentration and purity, and RNA integrity number (RIN). Samples with a RIN greater than seven underwent reverse transcription for cDNA synthesis. The University of Michigan Advanced Genomics Core measured gene expression using the Mouse Inflammation Taqman OpenArray (\#4475373), a high-throughput qRT-PCR of 648 inflammatory genes.

\section{Selection of genes for scaffold gene signature}

After OpenArray analysis, Cq values were analyzed in MATLAB to create a gene signature in a manner similarly to that used previously (Oakes et al, 2020; Morris et al, 2020a). First, any genes that were not detected in more than two mice in either group were removed from further analysis, and 549 of the 648 genes on the OA chip were used for this study. For some downstream analysis (that requires complete matrices such as singular value decomposition [SVD]), samples missing data for a particular gene were filled with the median of the entire dataset. Three reference genes were selected: $H m b s, U b c$, and $Y w h a z$ and $\Delta C_{q}$ values were calculated for each gene from the average of the reference genes for that sample. Fold change and $P$-values were calculated for diseased versus control samples for each gene. To create the scaffold gene signature, genes with a fold change $>1.5$ and $P<0.1$ were selected. This included: Ifng, Stat1, Ccr2, Irf7, Klrg7, Cx3cr1, Ccl4, II12b, Cxcl10, Ccl11, Cxcl14, Csf3, Tnfsf11, Nfatc4, F2r, Nox4, Cxcr4, Il6ra, Il18bp, Chi3l3, and Ccrl1/Ackr4.

\section{Gene signature scores and analysis}

Unsupervised hierarchical clustering was performed using the clustergram tool in MATLAB to plot dendrograms. This process allows clustering analysis of genes that cluster together as well as samples and can indicate if diseased scaffolds appear different from healthy. Next, computational approaches were applied to create two metrics determined from the scaffolds to indicate whether a mouse was diseased or healthy. We first created a score with an unsupervised technique, SVD using the svds function in MATLAB. Then we trained a bootstrap aggregated decision tree ensemble (Bagged Tree) with 100 learning cycles using MATLAB's fitcensemble function with the Bag method to classify samples as healthy or diseased. The bagged tree ensemble was fed the log2 transformed $\Delta C q$ values centered on the healthy controls as well as disease classification. This created our second score, a supervised machine learning metric that indicated the probability of disease.

\section{Single-cell RNA sequencing}

Scaffolds and human and mouse tissues were mechanically and enzymatically digested with collagenase $P(1 \mathrm{mg} / \mathrm{ml})$ and filtered through a $40-\mu \mathrm{M}$ mesh to obtain single cells. Dead cells were removed using MACS Dead Cell Removal Kit (Miltenyi Biotec Inc.). The single-cell cDNA libraries were prepared using the 10x Genomics platform at the University of Michigan, Advanced Genomics Core. All single-cell RNA sequencing samples were run using paired end 
50 cycle reads on either the HiSeq 4000 or the NovaSeq 6000 (Illumina) to a depth of 100,000 reads. Raw data were aligned to either mm10 or hg19 for mouse and human, respectively. Data were then filtered using Cellranger count V3.0.0 with default settings at the University of Michigan, Advanced Genomics Core. Downstream analysis was performed using R Studio V3.5.1 and R package Seurat V3.0. Batch correction across samples was performed using the R package Harmony V1.0 (https://github.com/immunogenomics/ harmony). Raw human data from the Steele et al study (Steele et al, 2020) are available at the National Institutes of Health (NIH) dbGaP database under the accession phs002071.v1.p1 and processed data are available at NIH Gene Expression Omnibus (GEO) database under the accession GSE155698. Raw and processed data for the iKras* (3 wk ON) samples are available under the accession GSE140628 (Zhang et al, 2020). Raw and processed data from this study are available at the NCBI's GEO database under the accession GSE158356.

\section{Statistics}

GraphPad Prism V7 software was used for graphical representation and statistical analysis. Two-tailed Student's t-tests were performed. A $P<0.05$ was considered statistically significant. Data are presented as means \pm standard error (SEM). Differential expression analysis in single-cell RNA sequencing data was performed using Wilcoxon rank sum test, with adjusted $P$-values for multiple comparisons.

\section{Data Availability}

Raw human data from the Steele et al study (Steele et al, 2020) are available at the $\mathrm{NIH}$ dbGaP database under the accession phs002071.v1.p1 and processed data are available at NIH GEO database under the accession GSE155698. Raw and processed data for the iKras* (3 wk ON) samples are available under the accession GSE140628 (Zhang et al, 2020). Raw and processed data from this study are available at the NCBI's GEO database under the accession GSE158356.

\section{Supplementary Information}

Supplementary Information is available at https://doi.org/10.26508/lsa. 202000935.

\section{Acknowledgements}

We thank Matthew Cochran and Terry Wightman at the Flow Cytometry Core at the University of Rochester Medical Center for CyTOF acquisition. We thank Kevin Brown and Vinicius Motta from Fluidigm for their assistance in CyTOF antibody panel design as well as downstream analysis in Cytobank. We thank Dr. Gregory Beatty, at the University of Pennsylvania, for the 7940b cell line. This work was made possible by the Advanced Genomics core at the University of Michigan. Funding: This project was supported by National Institutes of Health ( $\mathrm{NIH})$ / National Cancer Institute (NCI) grants R01CA151588, R01CA198074, and the American Cancer Society to M Pasca di Magliano. This work was also supported by the NIH U01CA224145 and University of Michigan Cancer Center Support Grant (P30CA046592), including an Administrative Supplement to HC Crawford and M Pasca di Magliano. F Bednar was funded by the Association of Academic Surgery Joel Roslyn Award. TL Frankel was funded by K08CA201581. SB Kemp was supported by NIH T32-GM113900 and $\mathrm{NCl}$ F31-CA247076. NG Steele, KL Donahue, and VR Sirihorachai were supported by T32-CA009676. NG Steele was funded by American Cancer Society Postdoctoral Award PF-19-096-01 and the Michigan Institute for Clinical and Healthy Research (MICHR) Postdoctoral Translational Scholar Program fellowship award. ES Carpenter was supported by the American College of Gastroenterology Clinical Research Award and by T32-DK094775. ZC Nwosu was supported by the Michigan Postdoctoral Pioneer Program, University of Michigan Medical School. A Rao and S The were funded by institutional startup funds from the University of Michigan, a gift from Agilent Technologies, NCI grant R37CA214955, and a Research Scholar Grant from the American Cancer Society (RSG-16-005-01). AH Morris received support from the Michigan Life Science Fellows award, NIH T32 grant DE007057-43, and National Institute of Biomedical Imaging and Bioengineering (NIBIB) grant K99EB028840. The funders had no role in study design, data collection and analysis, decision to publish, or preparation of the manuscript.

\section{Author Contributions}

SB Kemp: conceptualization, data curation, formal analysis, investigation, visualization, methodology, and writing-original draft, review, and editing.

NG Steele: data curation, formal analysis, methodology, and writing-review and editing.

ES Carpenter: data curation, formal analysis, methodology, and writing-review and editing.

KL Donahue: data curation and formal analysis.

GG Bushnell: data curation, methodology, and writing-review and editing.

AH Morris: data curation, formal analysis, methodology, and writing-review and editing.

$S$ The: data curation, formal analysis, and methodology.

SM Orbach: data curation, methodology, and writing-review and editing.

VR Sirihorachai: data curation and formal analysis.

ZC Nwosu: data curation, formal analysis, and writing-review and editing.

C Espinoza: data curation.

F Lima: data curation.

K Brown: data curation.

AA Girgis: data curation, formal analysis, and writing-review and editing.

$\checkmark$ Gunchick: resources and data curation.

Y Zhang: data curation, formal analysis, and writing-review and editing.

CA Lyssiotis: resources, methodology, and writing-review and editing.

TL Frankel: resources and methodology.

F Bednar: formal analysis and methodology.

A Rao: resources and methodology.

$\checkmark$ Sahai: resources, data curation, and methodology.

LD Shea: conceptualization, resources, and methodology.

HC Crawford: conceptualization, resources, funding acquisition, investigation, visualization, methodology, project administration, and writing-original draft, review, and editing. 
M Pasca di Magliano: conceptualization, resources, funding acquisition, investigation, visualization, methodology, project administration, and writing-original draft, review, and editing.

\section{Conflict of Interest Statement}

The authors declare that they have no conflict of interest.

\section{References}

Afshar-Kharghan V (2017) The role of the complement system in cancer. J Clin Invest 127: 780-789. doi:10.1172/jci90962

Aguado BA, Bushnell GG, Rao SS, Jeruss JS, Shea LD (2017) Engineering the pre-metastatic niche. Nat Biomed Eng 1: 0077. doi:10.1038/s41551-0170077

Aiello NM, Rhim AD, Stanger BZ (2016) Orthotopic injection of pancreatic cancer cells. Cold Spring Harb Protoc 2016: pdb.prot078360. doi:10.1101/pdb.prot078360

Azarin SM, Yi J, Gower RM, Aguado BA, Sullivan ME, Goodman AG, Jiang EJ, Rao SS, Ren Y, Tucker SL, et al (2015) In vivo capture and label-free detection of early metastatic cells. Nat Commun 6: 8094. doi:10.1038/ ncomms9094

Benoit ME, Clarke EV, Morgado P, Fraser DA, Tenner AJ (2012) Complement protein C1q directs macrophage polarization and limits inflammasome activity during the uptake of apoptotic cells. I Immunol 188: 5682-5693. doi:10.4049/jimmunol.1103760

Bettac L, Denk S, Seufferlein T, Huber-Lang M (2017) Complement in pancreatic disease-Perpetrator or savior? Front Immunol 8: 15. doi:10.3389/fimmu.2017.00015

Bonavita E, Gentile S, Rubino M, Maina V, Papait R, Kunderfranco P, Greco C, Feruglio F, Molgora M, Laface I, et al (2015) PTX3 is an extrinsic oncosuppressor regulating complement-dependent inflammation in cancer. Cell 160: 700-714. doi:10.1016/j.cell.2015.01.004

Bushnell GG, Hardas TP, Hartfield RM, Zhang Y, Oakes RS, Ronquist S, Chen H, Rajapakse I, Wicha MS, Jeruss JS, et al (2019) Biomaterial scaffolds recruit an aggressive population of metastatic tumor cells in vivo. Cancer Res 79: 2042-2053. doi:10.1158/0008-5472.can-18-2502

Bushnell GG, Orbach SM, Ma JA, Crawford HC, Wicha MS, Jeruss JS, Shea LD (2020) Disease-induced immunomodulation at biomaterial scaffolds detects early pancreatic cancer in a spontaneous model. Biomaterials 269: 120632. doi:10.1016/j.biomaterials.2020.120632

Clark CE, Hingorani SR, Mick R, Combs C, Tuveson DA, Vonderheide RH (2007) Dynamics of the immune reaction to pancreatic cancer from inception to invasion. Cancer Res 67: 9518-9527. doi:10.1158/00085472.can-07-0175

Collins MA, Bednar F, Zhang Y, Brisset JC, Galban S, Galban C), Rakshit S, Flannagan KS, Adsay NV, Pasca di Magliano M (2012a) Oncogenic Kras is required for both the initiation and maintenance of pancreatic cancer in mice. J Clin Invest 122: 639-653. doi:10.1172/jci59227

Collins MA, Brisset JC, Zhang Y, Bednar F, Pierre J, Heist KA, Galban C), Galban S, di Magliano MP (2012b) Metastatic pancreatic cancer is dependent on oncogenic Kras in mice. PLoS One 7: e49707. doi:10.1371/ journal.pone.0049707

Elyada E, Bolisetty M, Laise P, Flynn WF, Courtois ET, Burkhart RA, Teinor JA, Belleau P, Biffi G, Lucito MS, et al (2019) Cross-species single-cell analysis of pancreatic ductal adenocarcinoma reveals antigenpresenting cancer-associated fibroblasts. Cancer Discov 9: 1102-1123. doi:10.1158/2159-8290.cd-19-0094

Georgoudaki AM, Prokopec KE, Boura VF, Hellqvist E, Sohn S, Ostling J, Dahan R, Harris RA, Rantalainen M, Klevebring D, et al (2016) Reprogramming tumor-associated macrophages by antibody targeting inhibits cancer progression and metastasis. Cell Rep 15: 2000-2011. doi:10.1016/ j.celrep.2016.04.084

Gonzalez H, Hagerling C, Werb Z (2018) Roles of the immune system in cancer: From tumor initiation to metastatic progression. Genes Dev 32: 1267-1284. doi:10.1101/gad.314617.118

Hanahan D, Weinberg RA (2011) Hallmarks of cancer: The next generation. Cell 144: 646-674. doi:10.1016/j.cell.2011.02.013

Hingorani SR, Wang L, Multani AS, Combs C, Deramaudt TB, Hruban RH, Rustgi AK, Chang S, Tuveson DA (2005) Trp53R172H and KrasG12D cooperate to promote chromosomal instability and widely metastatic pancreatic ductal adenocarcinoma in mice. Cancer Cell 7: 469-483. doi:10.1016/ j.ccr.2005.04.023

Katzenelenbogen $\mathrm{Y}$, Sheban F, Yalin A, Yofe I, Svetlichnyy D, Jaitin DA, Bornstein C, Moshe A, Keren-Shaul H, Cohen M, et al (2020) Coupled SCRNA-seq and intracellular protein activity reveal an immunosuppressive role of TREM2 in cancer. Cell 182: 872-885.e19. doi:10.1016/j.cell.2020.06.032

Kinney JW, Bemiller SM, Murtishaw AS, Leisgang AM, Salazar AM, Lamb BT (2018) Inflammation as a central mechanism in Alzheimer's disease. Alzheimers Dement (N Y) 4: 575-590. doi:10.1016/ j.trci.2018.06.014

Kzhyshkowska J, Gratchev A, Goerdt S (2007) Human chitinases and chitinaselike proteins as indicators for inflammation and cancer. Biomark Insights 2: 128-146. doi:10.1177/117727190700200023

Lee JW, Stone ML, Porrett PM, Thomas SK, Komar CA, Li JH, Delman D, Graham K, Gladney WL, Hua X, et al (2019) Hepatocytes direct the formation of a pro-metastatic niche in the liver. Nature 567: 249-252. doi:10.1038/ s41586-019-1004-y

Lee PY, Wang JX, Parisini E, Dascher CC, Nigrovic PA (2013) Ly6 family proteins in neutrophil biology. J Leukoc Biol 94: 585-594. doi:10.1189/jlb.0113014

Li J, Byrne KT, Yan F, Yamazoe T, Chen Z, Baslan T, Richman LP, Lin JH, Sun YH, Rech AJ, et al (2018) Tumor cell-intrinsic factors underlie heterogeneity of immune cell infiltration and response to immunotherapy. Immunity 49: 178-193.e7. doi:10.1016/ j.immuni.2018.06.006

Liou GY, Bastea L, Fleming A, Doppler H, Edenfield BH, Dawson DW, Zhang L, Bardeesy N, Storz P (2017) The presence of interleukin-13 at pancreatic ADM/PanIN lesions alters macrophage populations and mediates pancreatic tumorigenesis. Cell Rep 19: 1322-1333. doi:10.1016/ j.celrep.2017.04.052

Long KB, Gladney WL, Tooker GM, Graham K, Fraietta JA, Beatty GL (2016) IFNgamma and CCL2 cooperate to redirect tumor-infiltrating monocytes to degrade fibrosis and enhance chemotherapy efficacy in pancreatic carcinoma. Cancer Discov 6: 400-413. doi:10.1158/21598290.cd-15-1032

Luo Y, Robinson S, Fujita J, Siconolfi L, Magidson J, Edwards CK, Wassmann K, Storm K, Norris DA, Bankaitis-Davis D, et al (2011) Transcriptome profiling of whole blood cells identifies PLEK2 and C1QB in human melanoma. PLoS One 6: e20971. doi:10.1371/journal.pone.0020971

Mauer J, Chaurasia B, Goldau J, Vogt MC, Ruud J, Nguyen KD, Theurich S, Hausen AC, Schmitz J, Bronneke HS, et al (2014) Signaling by IL-6 promotes alternative activation of macrophages to limit endotoxemia and obesity-associated resistance to insulin. Nat Immunol 15: 423-430. doi:10.1038/ni.2865

Melisi D, Niu J, Chang Z, Xia Q, Peng B, Ishiyama S, Evans DB, Chiao PJ (2009) Secreted interleukin-1alpha induces a metastatic phenotype in pancreatic cancer by sustaining a constitutive activation of nuclear factor-kappaB. Mol Cancer Res 7: 624-633. doi:10.1158/1541-7786.mcr08-0201

Mitchem JB, Brennan DJ, Knolhoff BL, Belt BA, Zhu Y, Sanford DE, Belaygorod L, Carpenter D, Collins L, Piwnica-Worms D, et al (2013) Targeting tumorinfiltrating macrophages decreases tumor-initiating cells, relieves 
immunosuppression, and improves chemotherapeutic responses Cancer Res 73: 1128-1141. doi:10.1158/0008-5472.can-12-2731

Molgora M, Esaulova E, Vermi W, Hou J, Chen Y, Luo J, Brioschi S, Bugatti M, Omodei AS, Ricci B, et al (2020) TREM2 modulation remodels the tumor myeloid landscape enhancing anti-PD-1 immunotherapy. Cell 182: 886-900.e17. doi:10.1016/j.cell.2020.07.013

Morris AH, Hughes KR, Oakes RS, Cai MM, Miller SD, Irani DN, Shea LD (2020a) Engineered immunological niches to monitor disease activity and treatment efficacy in relapsing multiple sclerosis. Nat Commun 11: 3871. doi:10.1038/s41467-020-17629-z

Morris AH, Orbach SM, Bushnell GG, Oakes RS, Jeruss JS, Shea LD (2020b) Engineered niches to analyze mechanisms of metastasis and guide precision medicine. Cancer Res 80: 3786-3794. doi:10.1158/00085472.can-20-0079

Murray PJ, Wynn TA (2011) Protective and pathogenic functions of macrophage subsets. Nat Rev Immunol 11: 723-737. doi:10.1038/ nri3073

Oakes RS, Bushnell GG, Orbach SM, Kandagatla P, Zhang Y, Morris AH, Hall MS, LaFaire P, Decker JT, Hartfield RM, et al (2020) Metastatic conditioning of myeloid cells at a subcutaneous synthetic niche reflects disease progression and predicts therapeutic outcomes. Cancer Res 80: 602-612. doi:10.1158/0008-5472.can-19-1932

Ohlund D, Handly-Santana A, Biffi G, Elyada E, Almeida AS, Ponz-Sarvise M, Corbo V, Oni TE, Hearn SA, Lee EJ, et al (2017) Distinct populations of inflammatory fibroblasts and myofibroblasts in pancreatic cancer. J Exp Med 214: 579-596. doi:10.1084/jem.20162024

Qian BZ, Pollard JW (2010) Macrophage diversity enhances tumor progression and metastasis. Cell 141: 39-51. doi:10.1016/j.cell.2010.03.014

Rao SS, Bushnell GG, Azarin SM, Spicer G, Aguado BA, Stoehr JR, Jiang EJ, Backman V, Shea LD, Jeruss JS (2016) Enhanced survival with implantable scaffolds that capture metastatic breast cancer cells in vivo. Cancer Res 76: 5209-5218. doi:10.1158/0008-5472.can-15-2106

Rhim AD, Mirek ET, Aiello NM, Maitra A, Bailey JM, McAllister F, Reichert M, Beatty GL, Rustgi AK, Vonderheide RH, et al (2012) EMT and dissemination precede pancreatic tumor formation. Cell 148: 349-361. doi:10.1016/j.cell.2011.11.025

Roszer T (2015) Understanding the mysterious M2 macrophage through activation markers and effector mechanisms. Mediators Inflamm 2015: 816460. doi:10.1155/2015/816460

Sanford DE, Belt BA, Panni RZ, Mayer A, Deshpande AD, Carpenter D, Mitchem JB, Plambeck-Suess SM, Worley LA, Goetz BD, et al (2013) Inflammatory monocyte mobilization decreases patient survival in pancreatic cancer: A role for targeting the CCL2/CCR2 axis. Clin Cancer Res 19: 3404-3415. doi:10.1158/1078-0432.ccr-13-0525

Shen ZT, Sigalov AB (2017) Novel TREM-1 inhibitors attenuate tumor growth and Prolong survival in experimental pancreatic cancer. Mol Pharm 14: 4572-4582. doi:10.1021/acs.molpharmaceut.7b00711

Siegel RL, Miller KD, Jemal A (2020) Cancer statistics, 2020. CA Cancer J Clin 70: 7-30. doi:10.3322/ caac.21590

Steele NG, Carpenter ES, Kemp SB, Sirihorachai VR, The S, Delrosario L, Lazarus J, Amir E-ad, Gunchick V, Espinoza C, et al (2020) Multimodal mapping of the tumor and peripheral blood immune landscape in human pancreatic cancer. Nat Cancer 1: 1097-1112. doi:10.1038/s43018020-00121-4

Stromnes IM, Brockenbrough JS, Izeradjene K, Carlson MA, Cuevas C, Simmons RM, Greenberg PD, Hingorani SR (2014) Targeted depletion of an MDSC subset unmasks pancreatic ductal adenocarcinoma to adaptive immunity. Gut 63: 1769-1781. doi:10.1136/gutjnl-2013-306271

Turnbull IR, Gilfillan S, Cella M, Aoshi T, Miller M, Piccio L, Hernandez M, Colonna M (2006) Cutting edge: TREM-2 attenuates macrophage activation. J Immunol 177: 3520-3524. doi:10.4049/jimmunol.177.6.3520

Wherry EJ, Ha SJ, Kaech SM, Haining WN, Sarkar S, Kalia V, Subramaniam S, Blattman JN, Barber DL, Ahmed R (2007) Molecular signature of CD8+ T cell exhaustion during chronic viral infection. Immunity 27: 670-684. doi:10.1016/j.immuni.2007.09.006

Wong KL, Tai JJ, Wong WC, Han H, Sem X, Yeap WH, Kourilsky P, Wong SC (2011) Gene expression profiling reveals the defining features of the classical, intermediate, and nonclassical human monocyte subsets. Blood 118: e16-e31. doi:10.1182/blood-2010-12-326355

Zhang R, Liu Q, Peng J, Wang M, Gao X, Liao Q, Zhao Y (2019) Pancreatic cancereducated macrophages protect cancer cells from complementdependent cytotoxicity by up-regulation of CD59. Cell Death Dis 10: 836. doi:10.1038/s41419-019-2065-4

Zhang Y, Lazarus J, Steele NG, Yan W, Lee HJ, Nwosu ZC, Halbrook CJ, Menjivar RE, Kemp SB, Sirihorachai VR, et al (2020) Regulatory T-cell depletion alters the tumor microenvironment and accelerates pancreatic carcinogenesis. Cancer Discov 10: 422-439. doi:10.1158/2159-8290.cd19-0958

Zhang Y, Velez-Delgado A, Mathew E, Li D, Mendez FM, Flannagan K, Rhim AD, Simeone DM, Beatty GL, Pasca di Magliano M (2017) Myeloid cells are required for $\mathrm{PD}-1 / \mathrm{PD}-\mathrm{L} 1$ checkpoint activation and the establishment of an immunosuppressive environment in pancreatic cancer. Gut 66: 124-136. doi:10.1136/gutjnl-2016-312078

Zhu Y, Herndon JM, Sojka DK, Kim KW, Knolhoff BL, Zuo C, Cullinan DR, Luo J, Bearden AR, Lavine KJ, et al (2017) Tissue-resident macrophages in pancreatic ductal adenocarcinoma originate from embryonic hematopoiesis and promote tumor progression. Immunity 47: 323-338.e6. doi:10.1016/j.immuni.2017.07.014

Zhu Y, Knolhoff BL, Meyer MA, Nywening TM, West BL, Luo J, Wang-Gillam A, Goedegebuure SP, Linehan DC, DeNardo DG (2014) CSF1/CSF1R blockade reprograms tumor-infiltrating macrophages and improves response to T-cell checkpoint immunotherapy in pancreatic cancer models. Cancer Res 74: 5057-5069. doi:10.1158/0008-5472.can-13-3723

Zimmerman KA, Bentley MR, Lever JM, Li Z, Crossman DK, Song CJ, Liu S, Crowley MR, George JF, Mrug M, et al (2019) Single-cell RNA sequencing identifies candidate renal resident macrophage gene expression signatures across species. J Am Soc Nephrol 30: 767-781. doi:10.1681/ asn.2018090931

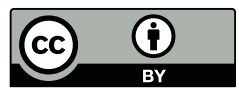

License: This article is available under a Creative Commons License (Attribution 4.0 International, as described at https://creativecommons.org/ licenses/by/4.0/). 